EFI-95-62

hep-th/9509149

\title{
String field theory in curved spacetime and the resolution of spacelike singularities
}

\author{
Albion Lawrence and Emil Martinec \\ Enrico Fermi Inst. and Dept. of Physics \\ University of Chicago, 5640 S. Ellis Ave., Chicago, IL 60637 USA
}

\begin{abstract}
We attempt to understand the fate of spacelike gravitational singularities in string theory via the quantum stress tensor for string matter in a fixed background. We first approximate the singularity with a homogeneous anisotropic background and review the minisuperspace equations describing the evolution of the scale factors and the dilaton. We then review and discuss the behavior of large strings in such models. In a simple model which expands isotropically for a finite period of time we compute the number density of strings produced by quantum pair production and find that this number, and thus the stress tensor, becomes infinite when the Hubble volume of the expansion exceeds the string scale, in a manner reminiscent of the Hagedorn transition. Based on this calculation we argue that either the region near the singularity undergoes a phase transition when the density reaches the order of a string mass per string volume, or that the backreaction of the produced string matter dramatically modifies the geometry.
\end{abstract}

\section{Introduction}

Infinities and singularities in physical theories are signals that the theory in question is inconsistent or incomplete, and force physicists to uncover deeper structures. Much of twentieth century physics has arisen from such singularities; two notable examples are the ultraviolet catastrophes of classical thermodynamics which helped drive the development of quantum mechanics, and the infinities in field theory loop calculations which helped drive the development and understanding of the renormalization group.

Among the theories which we currently use to describe the world, general relativity remains doubly afflicted: at the quantum level it is nonrenormalizable, and at the classical level it contains singularities, as demonstrated by the wellknown singularity theorems of Hawking and Penrose (Penrose 1965, Hawking 
1967, Hawking and Penrose 1970). With both of these singularities we are faced with the problem of predictability. Nonrenormalizability tells us that there must be a very different description of the world at the Planck scale, but gives no clue as to what that description might be. Classical spacetime singularities defined via geodesic incompleteness tell us that in finite proper time, we will reach regions of spacetime beyond which we cannot evolve the dynamical equations of the theory. Of course, if we attempt to cure this classical problem by quantizing the theory, we must contend with the nonrenormalizability of the theory.

One subject of active research which may force physicists to face these two problems directly is the apparent evaporation of black holes via quantum particle production (Hawking 1974). It is our belief that to understand the outcome of the Hawking process we must eventually understand the physics near the singularity. In particular this seems to be the primary lesson taught by the study of two-dimensional dilatonic black holes; at some point the singularity and the apparent horizon coincide and one needs some prescription for dealing with the singularity at this point in order to understand the final state of the black hole (see Strominger 1995 and references therein). To date there have been several proposals for the what endpoint the of black hole evaporation process might bef (for critical reviews and references see Banks 1994 and Strominger 1995); many of these proposals rely on low-energy physics up to the point that the black hole reaches the Planck mass, and then propose plausible consistent completions of the evaporation process, without recourse to a Planck-scale theory. Nonetheless, it remains to be shown explicitly that any of these proposals are the result of a complete theory of quantum gravity.

Thus, if we claim to understand quantum gravity, we must understand and remove the singularities that seem to plague it. This is hardly a new enterprise, and while we are not capable of producing a full history of previous attempts to do so, one may divide the attempts into two categories:

- Evasion of some tenet of the singularity theorems

- Searching for a breakdown of the notion of classical spacetime in a region of strong curvature, via quantum mechanics or string theory

The most natural way to avoid the singularity theorems is to violate the condition

$$
\mathcal{R}_{a b} \xi^{a} \xi^{b} \geq 0,
$$

(where $\mathcal{R}_{a b}$ is the Ricci tensor and $\xi$ is a timelike or null vector field depending on which theorem one appeals to); the other conditions involve global hyperbolicity of the spacetime (although this condition is non-essential), the lack of closed timelike loops, and the existence of trapped surfaces (which occurs given a

\footnotetext{
1 "The number of very good physicists who have expressed fairly definitive opinions about the resolution of the Hawking puzzle is smaller than the number of definitive opinions they have expressed." (Banks 1994)
} 
sufficient concentration of matter (Schoen and Yau 1983)), and are physically too attractive to casually dismiss. The singularity theorems are based on feeding Equation (11) back into the Raychaudhuri equation; in general relativity one can then use Einstein's equations to relate Equation (1D) to the strong or the null energy conditions. To avoid the singularity theorems one must then either violate the appropriate energy conditions, or suitably modify Einstein gravity so that Equation (1) is violated. In particular, quantum fields violate the energy conditions (Epstein et.al. 1965).

In the long run simply adding terms to Einstein's equations or relaxing energy conditions may not seem entirely satisfactory: we know that as we approach large field gradients close to the singularity, quantum geometry and (sub)Planckian physics come into play. Still, at this point the burden is on the theorist to explain what exactly quantum geometry is, and then to show that quantum mechanics combined with one's favorite choice of a Planck scale theory is not also singular, especially given the nonrenormalizability of Einstein gravity.

In this paper we would like to examine gravitational singularities using quantum string theory. To do this we should redefine singularities in terms of extended objects and quantum mechanics; we can do this with fairly natural extensions of geodesic completeness. Classically, we require that the solutions to the string equations of motion never cause the string to leave the spacetime in finite proper worldsheet distance. Quantum mechanically, we require that the first-quantized wave operator be well-defined and Hermitian everywhere. With these definitions we can discuss three types of singularities: singular sources of classical fields (i.e. charged point particles), timelike singularities, and spacelike singularities occurring (in a suitably defined coordinate system) at some point in time.

Singular classical sources are easily dealt with: they arise generally from considering particles as point-like objects. In a first-quantized theory quantum mechanics smears out the wave function of a test particle enough to avoid any pathology at the source, so long as the singularity in the potential is weaker than $1 / r^{2}$ (which is the case for the known forces of nature.) In quantum field theory calculations, we get divergences from large loop momenta and from small impact parameters. These are results of not properly regulating the theory at short distances.

Timelike singularities, of which the aforementioned singularities are a subset, are also not generically troublesome. It is strongly believed that string theory has a minimum distance and thus soft high-energy behavior, due to the extended nature of the fundamental objects. This has been shown to resolve many potential timelike singularities in the theory, most notably in amplitudes for high-energy scattering (Gross and Mende 1987, 1988), orbifold singularities (Dixon et.al. 1985, 1986), and singularities arising from the degeneration of Kähler structures in Calabi-Yau manifolds (Aspinwall et.al. 1993 and 1994, Witten 1993). This good behavior arises from the apparent lack of any opera- 
tive definition of a point in string theory: no point on a given string can carry a finite fraction of the string's energy, so that any momentum transfer in a stringy scattering process is shared by the entire string. We should note that we do not want to smooth out all timelike singularities. Horowitz and Myers (1995) have argued that any stable theory with Einstein gravity as a low-energy limit must have solutions with timelike singularities arising from singular initial conditions; in particular, a solution which looks like a negative-mass Schwarzschild solution at large distances should be singular even in a full theory of quantum gravity (remember that the total mass is measured at infinity, where only the Einstein term in the effective action will contribute). If we cannot throw away such solutions the theory becomes unstable since there is no stable ground state, i.e. empty space decays by pair-producing these negative-energy lumps (Horowitz and Myers, 1995).

Spacelike singularities pose a genuinely serious and confusing dilemma, as they occur entirely because of the universally attractive nature of gravity past the event horizon, where the causal structure forces classical matter to collapse completely. These facts seem to be independent of the ultraviolet nature of the theory. So, while the singularity is an ultraviolet object and perhaps will be resolved by physics similar to that which resolves timelike singularities, their inevitable nature and the fact that they arise from a rather soft attractive force begs for a careful examination. At any rate it is safe to say that we do not have as much control over these singularities in string theory as we have over the known timelike singularities, and so those of us who hope that string theory describes nature must still confront spacelike singularities.

The first words uttered by string theorists when faced with singular backgrounds is that they are simply signs that the expansion of the spacetime effective action in powers of $\ell_{\text {curv }} / \ell_{\text {str }}$, where $\ell_{\text {curv }}$. is the characteristic length over which the curvature changes, breaks down. Yet there are known exact conformal theories that possess spacelike singularities, the best known example being the 2-dimensional $S L(2, \mathcal{R}) / U(1)$ black hole, (Witten 1991). One might then appeal to string loop corrections to the $\beta$-functions (Lovelace 1986, Fischler and Susskind 1986, Callan et.al. 1987). While we will argue below that string loop effects are part of the story, we should be careful. For example, there are gravitational plane-wave backgrounds with null singularities, for which string propagation is singular (Horowitz and Steif 1990a and 1990b, de Vega and Sanchez 1992). These backgrounds correspond to exact conformal theories at tree level (Amati and Klimcik 1989, Horowitz and Steif 1990a) because all interesting counterterms that one might produce from the Riemann tensor, the dilaton, or the antisymmetric tensor field, will vanish; for the same reason loop corrections to the background will also vanish. However, it should be noted that these null singularities by definition do not arise from nonsingular initial conditions. We should also recall the arguments of Horowitz and Myers (1985) mentioned above: if corrections to the low energy effective action generically tame singular backgrounds, we should worry that the theory is unstable. 
The aforementioned string theorist would next point out that corrections to the effective action are not the end of the story; for example, strings propagating in orbifold geometries are quantum mechanically well behaved at the orbifold points. The lesson here is that we should relax our definition of singularities somewhat, and define them as regions of the background for which the wave operator for string theory becomes undefined, thus properly including orbifold geometries and some degenerations of Kähler geometries as nonsingular backgrounds. This definition is a natural extension of geodesic completeness as it defines a singularity as the point at which predictability, here defined as the ability to evolve quantum wave functions, breaks down. This relaxed definition does not save us from spacelike singularities. For example, the $L_{0}$ operator in the $S L(2, \mathcal{R}) / U(1)$ black hole background becomes ill-defined at the singularity: string wave functions (i.e. tachyon vertex operators) blow up at the singularity (Dijkgraaf et.al. 1992). In order to support our claim that $\alpha^{\prime}$ corrections and the properties of quantum wave functions in string theory do not in general save us from spacelike singularities, we will in the appendix review arguments which indicate that the structure of higher-dimensional black hole singularities is described by a perturbation of the $S L(2, \mathcal{R}) / U(1)$ black hole. Note also that the caveats of Horowitz and Myers (1995) apply to quantum mechanical smoothing as well.

None of this is to say that there is no solution to the problem of spacelike singularities in string theory, or that the answer does not involve the shortdistance properties of string theory; rather, we simply wish to emphasize that the problem has not been solved and that it requires some care. In the light of these arguments, this paper is an attempt to reach a primitive understanding of the quantum dynamics of string field theory in singular backgrounds, as a precursor to understanding the full quantum evolution of the background. We will make this attempt by first examining the spacetime background near the singularity and noting its generic and essential features, and then by examining string propagation in singular backgrounds, and finally by trying to crudely understand string field theory in a simplified spacetime background for which the scale factor changes over a brief period of time. We will find that the density of produced strings in a given state is exponentially suppressed by the mass of that state times the inverse rate of expansion, $\rho^{-1}$, so that when this rate becomes as large as $\ell_{\text {str }}^{-1}$, the density of states overwhelms the suppression and an infinite number of highly energetic long strings are produced. One may object that it is not legitimate to quantize string field theory in a classical background spacetime without explicitly taking backreaction into account from the start; however, since string theory has an as adjustable dimensionless parameter the ratio $\ell_{s t r} / \ell_{p l}$ far from the black hole, we may make this parameter small and see if string theory resolves the singularity in the weakly coupled region where we have some hope of knowing what to do. Our result indicates a sort of paradox, of which we see two possible resolutions: either stringy backreaction must smooth the singularity, or there is a Hagedorn-like phase transition as the 
density of produced strings becomes comparable to a string mass per unit string volume. In the former case we will suggest that the combination of an equation of state for string matter derived by Gasperini et.al. (1991a,b) and the effects of the dilaton (Tseytlin and Vafa 1992) potentially cause spacetime to become non-singular. Without thinking too much, one could imagine a few scenarios. For example, the spacetime could stabilize as expansion or contraction cease, with the dilaton kinetic energy balancing the produced energy density as the spacetime runs to strong coupling. Another possibility is that the singular region relaxes to some de-Sitter like phase, as suggested by Frolov et.al. (1990) (see also Poisson and Israel 1988) for the case of particle field theory, based on a limitingcurvature hypothesis for quantum gravity. One of us (Martinec 1995) proposed a realization of this latter possibility by appealing to T-duality: this paper arose from that work, although we no longer feel that the duality properties of string theory are necessarily relevant for our purposes. Our arguments will be crude and will ignore many issues such as the conformal invariance of the background, but we will argue that we have captured the physics of the effects we are interested in and that what we have ignored will not invalidate our results.

Since our proposals depend on the time-dependent nature of the singularities we are interested in, they may not apply to timelike singularities; the sorts of timelike singularities which Horowitz and Myers (1995) discuss may remain singular as required for the stability of flat space. As a limiting case, the singular null-fronted plane wave spacetimes described by Horowitz and Steif (1990) and de Vega and Sanchez (1992) will have no particle creation (and no string creation) due to the presence of a null Killing vector, so that in this borderline case the singularity is untamed by our scenarios.

The remainder of the paper shall be organized as follows: in Section 2 we will review features of singular backgrounds in general relativity and string theory. In the case of string theory, we will examine the cosmological equations of Tseytlin and Vafa (1992) with respect to various phenomenological energymomentum tensors that one may imagine, and discuss the singularities that may arise in these equations. In Section 3 we will describe string propagation in expanding and/or contracting backgrounds, reviewing the work of Gasperini, Sanchez, and Veneziano (Sanchez and Veneziano 1990, Gasperini et.al. 1991a,b). We will use a similar approximation to examine the propagation of large strings in a temporarily expanding model. We will also discuss the equation of state for string matter in these models. In Section 4 we will discuss free quantum string field theory in curved backgrounds, and describe techniques for computing approximations to the rate of string production in such backgrounds. In Section 5 we will apply these techniques to a specific isotropically expanding model and find the aforementioned singularity in the rate of string production. In section 6 we will discuss how one might extend this calculation to an anisotropic model and what features one might look for; we will also discuss how the produced matter, given the equation of state discussed in section 3 , might affect the 
singularity. Section 7 will contain our conclusions and further speculations. Appendix A will discuss how one might relate the $S L(2, \mathcal{R}) / U(1)$ black hole to the Schwarzschild solution.

\section{Classical backgrounds in general relativity and string theory}

Belinskii, Khalatnikov, and Lifshitz (Lifshitz and Khalatnikov 1963, Belinskii et.al. 1970, 1982) have argued that the spacetime metric close to a spacelike singularity can be written as

$$
d s^{2}=-d t^{2}+\left(t^{2 p_{1}} l_{\alpha} l_{\beta}+t^{2 p_{2}} m_{\alpha} m_{\beta}+t^{2 p_{3}} n_{\alpha} n_{\beta}\right) d x^{\alpha} d x^{\beta},
$$

where $p_{i}, l, m$, and $n$ are functions of the spatial coordinates only, and the $p_{i}$ satisfy the equations

$$
\sum_{i=1}^{3} p_{i}=\sum_{i=1}^{3} p_{i}^{2}=1
$$

which are identical to the restrictions on the spatially independent exponents $p_{i}$ in a vacuum Kasner spacetime:

$$
d s^{2}=-d t^{2}+\sum_{i=1}^{3} t^{2 p_{i}}\left(d x^{i}\right)^{2} .
$$

Equation (2) is found by expanding Einstein's equations in a power series in $t$, assuming a power law singularity in the metric, with the leading exponents as shown: one finds that contributions from the intrinsic curvature of the spatial slices are subleading as compared to their extrinsic curvature (though there is the requirement that, if $p_{1}$ is the negative exponent, $\vec{l} \cdot \nabla \times \vec{l}=0$, which means that we may use the direction specified by $l$ as a global coordinate). This means that the spacetime is "velocity-dominated" in this region. If we look on a reasonably small spatial slice, the metric should look like the Kasner metric. The form (2) is certainly what we find near the Schwarzschild singularity: the metric inside the horizon $\left(r<r_{h}\right)$ for a D-dimensional Schwarzschild black hole is

$$
\begin{aligned}
d s^{2} & =-\left[\left(\frac{r_{h}}{r}\right)^{D-3}-1\right]^{-1} d r^{2}+\left[\left(\frac{r_{h}}{r}\right)^{D-3}-1\right] d t^{2}+r^{2} d \Omega_{D-2}^{2} \\
& \sim-\left(\frac{r}{r_{h}}\right)^{D-3} d r^{2}+\left(\frac{r_{h}}{r}\right)^{D-3} d t^{2}+r^{2} d \Omega_{D-2}^{2} .
\end{aligned}
$$


If we write $T=-\left[2 r_{h} /(D-1)\right]\left(r / r_{h}\right)^{(D-1) / 2}$, and $\rho=t$, we can rewrite the most singular part of the metric as

$$
d s^{2}=-d T^{2}+\left[\frac{(D-1) T}{2 r_{h}}\right]^{-2(D-3) /(D-1)} d \rho^{2}+r_{h}^{2}\left[\frac{(D-1) T}{2 r_{h}}\right]^{4 /(D-1)} d \Omega_{D-2}^{2}
$$

Note that in four dimensions the relations (3) are satisfied. We conclude that the essential physics of the velocity-dominated singularity can be captured by looking at homogeneous anisotropic spacetimes.

Einstein's equations are contained in the lowest order $\beta$-function equations of string theory (Lovelace 1986, Callan et.al. 1985, Fradkin and Tseytlin 1985, Sen 1985); one does not expect the Schwarzschild solution to describe the geometry as one approaches the singularity, where higher order terms in the $\beta$-function equations become important. Nonetheless it is worth repeating that there are exact conformal theories which contain spacelike singularities, including the $S L(2, \mathcal{R}) / U(1)$ black hole (Witten 1991), various four dimensional black hole backgrounds consisting of the tensor product of this theory with other conformal field theories (Giddings et.al. 1993, 1994, Johnson 1994, Lowe and Strominger 1994), and a four dimensional cosmological model, with initial and final singularities, constructed as a $\mathrm{c}=4 \mathrm{WZW}$ model (Nappi and Witten 1992). Furthermore, there are some indications that the $S L(2, \mathcal{R}) / U(1)$ black hole may be relevant for understanding the background near the singularity in higher-dimensional spacetimes; we will review these indications in the appendix.

We will start with something rather simpler than these exact conformal field theories, looking instead for homogeneous solutions to the one-loop beta functions for the metric coupled to the dilaton $\phi$ and matter with central charge

$$
c=\frac{2}{3}\left(d_{\text {crit }}-D-N_{\text {compact. }}\right),
$$

where $d_{\text {crit }}$ is the critical dimension of the string theory, $D$ is the number of macroscopic dimensions, and $N_{\text {compact. }}$ is the number of compactified dimensions. If we write the string frame metric as

$$
d s^{2}=-d t^{2}+\sum_{i} e^{2 \lambda_{i}(t)}\left(d x^{i}\right)^{2},
$$

then the $\beta$-function equations can be written as (Tseytlin and Vafa 1992)

$$
\begin{aligned}
& c-\sum_{i=1}^{D-1}\left(\dot{\lambda}_{i}\right)^{2}+\left(2 \dot{\phi}-\sum_{i=1}^{D-1} \dot{\lambda}_{i}\right)^{2}=\rho e^{a \phi} \\
& \ddot{\lambda}_{k}-\left(2 \dot{\phi}-\sum_{i=1}^{D-1} \dot{\lambda}_{i}\right) \dot{\lambda}_{k}=e^{a \phi} \frac{p_{k}}{2} \\
& 2 \ddot{\phi}-\sum_{i=1}^{D-1} \ddot{\lambda}_{i}-\sum_{i=1}^{D-1} \dot{\lambda}_{i}^{2}=e^{a \phi} \frac{\rho}{2} .
\end{aligned}
$$


Here $\rho$ is the energy density, and $p_{k}$ is the pressure in the direction of $x^{k}$ : these terms come from adding a phenomenological matter action to the low energy effective action that can be derived from the usual $\beta$-function equations. We may also include the effect of potential terms, such as that coming from a dilaton potential, in $\rho$ and $p$. In general $a$ will depend on how the matter couples to the dilaton. For a classical fundamental string source, the total action without the antisymmetric tensor field is (Dabholkar and Harvey 1989, Dabholkar et.al. 1990)

$$
\begin{aligned}
S= & \frac{1}{16 \pi G} \int d^{D} x \sqrt{g} e^{-2 \phi}\left\{\mathcal{R}+4(\nabla \phi)^{2}\right\} \\
& -\frac{1}{2 \pi \alpha^{\prime}} \int d^{2} \sigma\left\{\sqrt{h} h^{\alpha \beta} g_{\mu \nu} \partial_{\alpha} X^{\mu} \partial_{\beta} X^{\nu}+\frac{\alpha^{\prime}}{2} \mathcal{R}^{(2)} \phi(X)\right\} \\
& =S_{\text {spacetime }}+S_{\text {worldsheet }}[X]
\end{aligned}
$$

where $\mathcal{R}$ is the spacetime curvature, $\mathcal{R}^{(2)}$ is the worldsheet curvature, and $X(\sigma, \tau)$ is the location of the worldsheet. Here the fundamental string couples as a classical source, and thus lacks the $\exp (-2 \phi)=1 / g_{\text {str }}^{2}$ factor. (We will discuss the justification for Equation (10) at the end of the following section.) Naturally, for multiple strings, one may sum the worldsheet action over the different sources, making the replacement

$$
S_{\text {worldsheet }}[X] \longrightarrow \sum_{i} S_{\text {worldsheet }}\left[X_{i}\right]
$$

in Equation (10). Now if the stress tensor arises from a gas of classical strings, we expect that $a=2$ due to the lack of a dilaton prefactor in the worldsheet action (Veneziano 1991, Tseytlin 1992). We will also get different values of $a$ depending on whether the matter comes from higher loop effects and so on (Tseytlin 1992). In the particle production scenario we are proposing, the number density will be proportional to $\exp (-$ const. $/ \dot{\lambda})$, so that we will set $a=2$ and let $\rho=\rho(\phi, \lambda, \dot{\lambda})$ and $p=p(\phi, \lambda, \dot{\lambda})$. It is worth noting that if we set the dilaton to be constant then Equation (9) has solutions only if

$$
\rho=\sum_{i=1}^{D-1} p_{k}+2 c .
$$

In particular, for $c=0$ a constant dilaton and a homogeneous metric requires a radiation-like equation of state.

Many solutions to Equation (9) have been described by Tseytlin (1992): we would like to prepare for later discussions by sketching a few possible solutions. Define a new field $\varphi=2 \phi-\sum_{i=1}^{n} \lambda_{i}$, and set $a=2$; then Equation (9) becomes

$$
c-\sum_{i=1}^{n} \dot{\lambda}_{i}^{2}+\dot{\varphi}^{2}=e^{2 \phi} \rho
$$




$$
\begin{aligned}
& \ddot{\lambda}_{k}-\dot{\varphi} \dot{\lambda}_{k}=\frac{1}{2} e^{2 \phi} p_{k} \\
& \ddot{\varphi}-\sum_{i=1}^{n} \dot{\lambda}_{i}^{2}=\frac{1}{2} e^{2 \phi} \rho
\end{aligned}
$$

(Tseytlin and Vafa, 1992). The first equation may be used to solve for $\dot{\varphi}$, so that the second equation becomes

$$
\ddot{\lambda_{k}}=\frac{1}{2} p_{k} e^{2 \phi} \pm \dot{\lambda}_{k} \sqrt{\rho e^{2 \phi}+\sum_{i=1}^{D} \dot{\lambda}_{i}^{2}-c}
$$

With $\rho, p=0$ the solution will be a generalization of the Kasner solution with

$$
\begin{aligned}
\varphi(t) & =\varphi_{0}-\ln \left(t_{c}-t\right) \\
\lambda_{i}(t) & =\lambda_{i, 0}+q_{i} \ln \left(t_{c}-t\right) \\
\sum_{i=1}^{D-1} q_{i}^{2} & =1
\end{aligned}
$$

(Tseytlin 1992). Note that this corresponds to the + branch of the square root in Equation (14). We wish to match this solution to the Schwarzschild black hole near the singularity, which as we have argued can be approximated by a Kasner metric. Thus, we would start with the solution to Equation (15) corresponding to $\dot{\phi}=0$. At this point we assume that particle production begins; what happens next depends on the details of the equation of state. In particular note that in order to turn around runaway expansion or contraction it is necessary that the pressure term become sufficiently large and negative for rapidly expanding spacetimes and sufficiently large and positive for rapidly contracting spacetimes. We will argue in the next section that string theory gives us exactly this sort of behavior.

It is worth repeating here a point made by Tseytlin and Vafa (1992). In Einstein gravity we are used to any energy density at all contributing to the expansion of the universe, at least for a spatially flat cosmology where $\dot{\lambda}^{2}=$ $(8 \pi G / 3) \rho$. We are arguing that a negative pressure may in fact provide a drag on the expansion. The difference is the presence of the dilaton, which is an embarrassment for phenomenology but may be a blessing when dealing with singularities. Note also that runaway expansion or even a sufficient ammount of (positive) energy density will cause the dilaton to run to infinite coupling in finite time, making string loops especially important. On the other hand, sufficient contraction and insufficient energy density can cause the dilaton to run to weak coupling. 


\section{String matter as a source for the field equa- tions}

Sanchez and Veneziano (1990) demonstrated that in a large class of inflationary spacetimes (with a constant dilaton), certain string modes became unstable and begin growing with the scale factor. They argued that this happens when the string size becomes larger than the horizon size of the expansion, so that different parts of the string cease to communicate with each other. They showed that this occured potentially with power-law expansion and de Sitter expansion, and inevitably with superinflationary expansion. Gasperini et.al. (1991a) studied the approximate behavior of large strings in the homogeneous, isotropic metric

$$
d s^{2}=\frac{1}{\eta^{2 \alpha}}\left(-d \eta^{2}+\sum_{i=1}^{D-1}\left(d x^{i}\right)^{2}\right)
$$

which describes power-law inflation for $\alpha>1$, de Sitter inflation for $\alpha=1$, and superinflation for $0<\alpha<1$. They found that for sufficiently large strings, the conformal time $\eta$ was proportional to the worldsheet time $\tau$ in conformal gauge, and that one could expand the solution to the classical equations of motion and Virasoro constraints in a power series expansion in the worldsheet time $\tau$ and thus show that a consistent lowest-order solution to the equation of motion plus constraints is

$$
\begin{aligned}
& \vec{X}=\vec{X}(\sigma)+\mathcal{O}\left(\tau^{2}\right)+\ldots \\
& \eta=\tau \sqrt{\vec{X}^{2}(\sigma)}+\mathcal{O}\left(\tau^{2}\right),
\end{aligned}
$$

where $0<\sigma<2 \pi$ is the worldsheet spatial coordinate. Note that in this

approximation, $\vec{X}^{\prime} \gg \dot{\vec{X}}$, and $\dot{\eta} \gg \eta^{\prime}$ as $\tau, \eta \longrightarrow 0$ (where the dot and prime refer to worldsheet time and space derivatives respectively), which is what one would expect from a large, slow string. In a follow-up paper (Gasperini et.al. 1991b), they showed that in rapidly contracting spacetimes one finds unstable behavior for which the worldsheet time is proportional to the comoving time $t$; the unstable behavior is characterized by $\vec{X}^{\prime} \ll \dot{\vec{X}}$, and $\dot{t} \gg t^{\prime}$. (the former condition came from a term of the form $\tau^{1-\alpha} \vec{X}_{2}$, where $\alpha<1$, which has a large time derivative as $\tau \rightarrow 0$.)

Instead of repeating the calculations described above, we shall instead perform a similar calculation to find the approximate behavior of very large strings in a temporarily expanding universe by using the string size, rather than the temporal distance to an infinite scale factor, as the expansion parameter. This will give us some intuition for the calculations we will perform in the following two sections. Consider an isotropically expanding or contracting universe with metric

$$
d s^{2}=C(\eta)\left(-d \eta^{2}+d \vec{X}^{2}\right) .
$$


The classical equations of motion and the classical constraints in conformal gauge for a string in this spacetime are:

$$
\begin{aligned}
& -\ddot{\eta}+\eta^{\prime \prime}-\frac{C_{\eta}}{2 C}\left[\dot{\eta}^{2}-\eta^{\prime 2}+\dot{\vec{X}}^{2}-\vec{X}^{\prime 2}\right]=0 \\
& -\ddot{\vec{X}}+\vec{X}^{\prime \prime}-\frac{C_{\eta}}{C}\left[\dot{\eta} \dot{\vec{X}}-\eta^{\prime} \vec{X}^{\prime}\right]=0 \\
& C\left[\dot{\eta}^{2}+\eta^{\prime 2}-\dot{\vec{X}}^{2}-\vec{X}^{\prime 2}\right]=0 \\
& C\left[-\dot{\eta} \eta^{\prime}+\dot{\vec{X}} \cdot \vec{X}^{\prime}\right]=0 .
\end{aligned}
$$

The first two equations are the equations of motion and the last two are the Virasoro constraints. We wish to expand these equations in a power series in the size of the string. Let $L$ be the dimensionless length, i.e. the full length of the string divided by $\ell_{s t r}$. Following Gasperini et.al. (1991a), we assume that the time dependence of the spatial coordinates is of lower order. We thus expand $\vec{X}$ in the series:

$$
\vec{X}(\tau, \sigma)=L \vec{X}_{0}(\sigma)+\sum_{n=1}^{\infty} L^{1-n} \vec{X}_{n}(\tau, \sigma),
$$

where the $\vec{X}_{n}$ are all of order $\ell_{s t r}$. We must be a little careful about the the worldsheet time interval we are interested in. As we will see, the lowest order solution to the constraints is $\eta_{0}=\tau L \sqrt{\vec{X}_{0}^{\prime 2}}$; we will be interested below in universes which expand for a target space time interval of order $\rho^{-1}$, where $\ell_{s t r} L / \rho \gg 1$, so that the worldsheet time interval of interest will be of order $1 / L$. In order to keep track of these factors explicitly let us define $s=L \tau$, so that $s$ will be of order 1 in this expansion. Then we may expand $\eta$ in the series

$$
\eta=\sum_{n=0}^{\infty} \eta_{n}(\tau, \sigma) L^{-n}
$$

In general we should note that we must expand $C(\eta)$ in a power series in $1 / L$ as well, though this will not show up in the lowest order equations. These lowest order equations are

$$
\begin{aligned}
& \ddot{\eta}_{0}+\frac{C_{\eta}\left(\eta_{0}\right)}{2 C\left(\eta_{0}\right)}\left(\dot{\eta}_{0}^{2}+\dot{\vec{X}}_{1}^{2}-\vec{X}_{0}^{\prime 2}\right)=0 \\
& \ddot{\vec{X}}_{1}+\frac{C_{\eta}\left(\eta_{0}\right)}{C\left(\eta_{0}\right)} \dot{\eta}_{0} \dot{\vec{X}}_{1}=0 \\
& \dot{\eta}_{0}^{2}-\dot{\vec{X}}_{1}^{2}-\vec{X}_{0}^{\prime 2}=0 \\
& \dot{\vec{X}}_{1} \cdot \vec{X}_{0}^{\prime}=0
\end{aligned}
$$


where the dot denotes a derivative with respect to $s$. One may solve these equations for arbitrary $\vec{X}_{0}(\sigma)$ with $\dot{\vec{X}}_{1}=0$ (so that we may absorb $\vec{X}_{1}$ into $\vec{X}_{0}$ and thus set $\vec{X}_{1}=0$ ) and

$$
\begin{aligned}
& \dot{\eta}_{0}=\sqrt{\vec{X}_{0}^{\prime 2}} \text { or } \\
& \eta_{0}=s \sqrt{\vec{X}_{0}^{\prime 2}}=L \tau \sqrt{\vec{X}_{0}^{\prime 2}} .
\end{aligned}
$$

The interested reader may check these equations to the next order and verify that solutions exist given these first order solutions.

Note that in this case, just as in the case of the approach to the $\eta \rightarrow 0^{-}$in the work of Gasperini et.al. (1991), the comoving string coordinates are frozen to lowest order (the time derivatives of $\vec{X}$ are of order $1 / L$ ), so that the proper size of string will grow with the scale factor. We shall find that the same happens to the quantum wave functions below.

If a large number of such unstable strings are produced as we will claim, we would like to know their equation of state, i.e. what the spacetime stress-energy tensor is for this collection of strings. For a single macroscopic string source, the action for the background fields coupled to this source is given by Equation (10). The stress tensor is then found by taking the derivative of this action with respect to the spacetime metric in the usual manner (Dabholkar and Harvey 1989, Dabholkar et.al. 1990):

$$
\left.T_{\mu \nu}(x)=-\frac{\mu}{\sqrt{G}} \int d^{2} \sigma \sqrt{h} h^{\alpha \beta} \partial_{\alpha} X_{\mu} \partial_{\beta} X_{\nu} e^{\gamma \phi} \delta^{D}(X(\tau, \sigma)-x)\right)
$$

Using this ansätz for the stress tensor, Gasperini et.al. (1991b) show that the approximate equation of state for unstable strings shrinking with the scale factor in a contracting universe is

$$
\rho=\sum_{i=1}^{D-1} p_{i},
$$

which is the equation of state for photon-like particles; for unstable strings growing with the scale factor in an expanding universe (1991a) they find that

$$
\rho=-\sum_{i=1}^{D-1} p_{i}
$$

This is the sort of behavior we desired in the previous section, in order to remove the singularity, although for general anisotropic spacetimes we expect the equation of state to be more complicated.

The ansätz shown in Equation (10) for the coupling of a fundamental string source to the spacetime action still has the status of a conjecture, although there is evidence which supports it. Callan and Khuri (1991), Khuri (1993) and 
Gauntlett et.al. (1994) have found that using this expression and calculating the low-energy scattering of two such strings using collective coordinate methods, one can reproduce the scattering amplitudes found from the low-energy limit of the Virasoro-Shapiro amplitudes. Tseytlin (1990) has attempted to derive Equation (10) by summing up long thin handles on the string worldsheet (these are essentially wormholes on the worldsheet). This is a very attractive and probably correct answer but it requires that one make a connection between the spatial size of the macroscopic string and the dominance of "thin handles" in the integral over the moduli space of high-genus Riemann surfaces. We do not understand how to connect spacetime scales to scales in these moduli spaces, and believe this to be an interesting problem. Still, the intuition that large strings should be treated as sources of spacetime energy-momentum, and that this effect should arise from the summation of string loops describing the interaction of the macroscopic string with its surroundings, is physically fairly sound; thus, if the effects of these strings are significant, we know that the dynamics of string theory causes certain classes of loop diagrams to become important, so that we must at least modify the $\beta$-function equations appropriately. (Note that this is independent of another reason that loops might become important, namely that the dilaton may diverge near the singularity.) We shall comment on this point further in the conclusion.

\section{String field theory in curved spacetime}

\subsection{How do we evolve the background forward?}

Naturally, we would prefer to understand the full quantum mechanical behavior of string field theory near the singularity, where field strengths and quantum fluctuations are large; we could then evolve the string field theory wave functional corresponding to the black hole background from a point where we trust our classical understanding of the geometry to the dangerous region near the classical singularity. Unfortunately this computation would require a manifestly background independent quantum formulation of string field theory, not to mention a powerful computational control of that theory, both of which we lack. Even in the case of ordinary field theory, if we had a quantum theory of gravity, we would still have to contend with questions of interpreting the strongly fluctuating spacetime near the singularity. We will thus adopt the standard approach of computing the quantum stress tensor of matter in a fixed background, in order to use it as a source term for the $\beta$-function equations of string theory.

As we stated in the Introduction, this may be a reasonable approach for us. Since we are given as an adjustable parameter $\ell_{p l} / \ell_{s t r}$, we will perform our calculation in a regime where this ratio is small and we can trust the results of perturbative string theory; then, string physics becomes important well before 
quantum fluctuations of spacetime become important. This is not necessarily the end of the story; if the background is composed of strings, then at the string scale there is no clean separation between the background and the string fields we are quantizing on top of it.

In models with rapidly expanding directions there is another problem with keeping the background separate; if gravitons become unstable and grow with the scale factor, then the background gravitons become macroscopic strings and we must discuss the background as a soup of macroscopic strings. Perhaps there is some way of including this effect self-consistently to get some sort of modified $\beta$-function equations which are valid in these regimes. Based on our discussion of macroscopic strings in the previous section, this would amount to the inclusion of a certain class of loop corrections to the $\beta$-function equations. For the present, however, we will not attack this problem; rather, we will work in a background with a Hubble volume much larger than one in string units, and extrapolate the result to $r_{\text {Hubble }}=\ell_{\text {string. }}$. If we can do nothing else, we can at least argue that the above issues must eventually be addressed.

What we would like to calculate is the renormalized stress-energy tensor for string fields in a singular background. This is difficult in particle field theory, and should be at least as difficult in string field theory. As we have mentioned previously, in string theory we have at our disposal a few exact conformal field theories with spacelike singularities (Witten 1991, Giddings et.al. 1993,1994, Johnson 1994, Lowe and Strominger 1994, Nappi and Witten 1992). Since these examples correspond to gauged WZW models we might have some hope of calculating $\left\langle T^{\mu \nu}\right\rangle$ exactly. However, several problems arise. The most natural calculation to perform is that of the one-loop expectation value

$$
\left\langle T_{\text {spacetime }}^{\mu \nu}(x)\right\rangle=\frac{1}{\pi \alpha^{\prime} \sqrt{G(x)}}\left\langle\int d^{2} \sigma \eta^{\alpha \beta} \partial_{\alpha} X^{\mu} \partial_{\beta} X^{\nu} \delta(X(\tau, \sigma)-x)\right\rangle
$$

in the first-quantized picture (this expression is derived by taking the derivative of the first-quantized one-loop partition function - which should correspond to the spacetime one-loop effective action for the background fields (Fradkin and Tseytlin 1985)). For this calculation we must know the expectation value for the general off-shell graviton vertex operator, and so we require an off-shell understanding of the theory that we do not now possess. In addition, we must ensure that we are computing this expectation value (27) so that it corresponds to the appropriate second-quantized vacuum, which means we must find some sort of prescription for dealing with the poles in the string propagator (recall that in particle field theory the vacuum is defined by the Feynman propagator see Fulling's book (Fulling 1989) for a discussion). Finally, the quantization of strings propagating on non-compact WZW and gauged WZW models is not well understood. Given these problems, we will instead make a series of approximations in order to make the calculation tractable. First, we will ignore concerns about the conformal invariance of the background, which will allow us to work 
with simplified models of expanding spacetimes. Second, we will work only with the $L_{0}$ constraint on the string wave functions, rather than the infinite number of equations arising from the full Virasoro constraints. Third, we will set the spacetime conformal time $\eta$ to be a function solely of the worldsheet time $\tau$ in conformal gauge (otherwise, solving for the string configuration at a given $\eta$ becomes extremely difficult). Finally, since we do not know how to compute the string energy-momentum tensor directly, we will compute the number of strings produced and from that calculation determine the stress-energy tensor.

These approximations should not invalidate our results. First, we will be looking for semiclassical solutions to the string wave equation. As in the computation of the $\beta$-functions using the background field method, when expanding the first-quantized path integral of the string around a classical solution, violations of conformal invariance appear as logarithmic divergences in the quadratic fluctuation determinant. The effects we shall be interested in, namely the exponential dependence of the production amplitude on the energy, come from the classical term $\exp \left\{\left(i / \alpha^{\prime}\right) S_{\mathrm{cl}}\right\}$ of the wave function. Second, although we use only the $L_{0}$ constraints rather the full set of positive-mode Virasoro constraints, imposing the latter should simply have the effect of reducing the degrees of freedom of the strings that we produce. A useful analogy is the computation of Hawking radiation in QED; if we work in the Lorentz gauge and forget about the Gupta-Bleuler quantization procedure, we get the same basic physics out of the calculation, but we find that the calculated Hawking flux is too large by a factor of $D /(D-2)$, due to the effect of the unphysical timelike and longitudinal modes of the photon. A similar story should describe our calculation as well. Third, although setting $\eta=\eta(\tau)$ is not consistent with the full Virasoro conditions, as we have seen, an examination of Equation (19) shows that this ansätz is consistent with the $L_{0}$ constraints. Finally, although generally the concept of single particles or single strings is ambiguous in curved spacetime backgrounds, we will work with a spacetime in which string number does make sense, namely a temporarily expanding model which becomes flat Minkowski space in the far past and in the far future. Here we may sensibly calculate the number of strings using standard techniques and then simply multiply the number of strings in a given quantum mechanical state by the spacetime energy-momentum tensor of a string in that state, using the results described in the previous section, or some modification of them. Our methods will not be directly extendible to a calculation of the quantum stress tensor in a spacetime in which the future is a singularity rather than Minkowski space, but it will allow us to make some sensible statements about quantum string field theory in curved spacetime which should apply to the region we are interested in.

\subsection{Imposing the $L_{0}$ constraints}

We wish to calculate the number of different strings produced just as we would calculate the number of particles produced: by finding the solutions to the first- 
quantized relativistic wave equation which are purely positive frequency in the far past and looking for the coefficients of negative-frequency components in the far future. For string theory, the (linearized) wave equation is just the Virasoro condition:

$$
\begin{aligned}
& T_{++}+T_{--}=G_{\mu \nu}(X(\sigma))\left(\dot{X}^{\mu} \dot{X}^{\nu}+X^{\prime \mu} X^{\prime \nu}\right)=0 \\
& T_{++}-T_{--}=G_{\mu \nu} X^{\prime \mu} \dot{X}^{\nu}=0 .
\end{aligned}
$$

We wish to turn the Virasoro operators into functional differential operators acting on wave functionals $\Psi(X(\sigma))$. In curved space, there are subtleties even in the case of particle wave functions due to the necessity of defining the Hilbert space norms and the path integral measure in a covariant fashion (DeWitt 1957); we will assume the same story applies when we derive the string wave operator from the first-quantized path integral. The functional differential operator form of (28) should look like

$$
\begin{aligned}
& T_{++}+T_{--}= \\
& -\frac{1}{\sqrt{G(X(\sigma))}} \frac{\delta}{\delta X^{\mu}(\sigma)} \sqrt{G(X(\sigma))} G^{\mu \nu}(X(\sigma)) \frac{\delta}{\delta X^{\nu}(\sigma)}+ \\
& \quad G_{\mu \nu}(X(\sigma)) X^{\prime \mu} X^{\prime \nu}+\xi \mathcal{R} \\
& T_{++}-T_{--}=X^{\prime \mu} \frac{\delta}{\delta X^{\mu}},
\end{aligned}
$$

where $\mathcal{R}$ is the spacetime Ricci scalar and $\xi$ depends on whether the string is conformally, or otherwise, coupled (in other words, with what coefficient we multiply a term like $\mathcal{R}(X(\sigma))$ in the first-quantized string Lagrangian.) We prefer to remain agnostic as to how the string is coupled; at any rate, in the approximations we shall look at the curvature term will be of lower order and so we will ignore it.

There are several issues we must deal with before using Equation 29 as a wave operator. First, it must be normal ordered. Secondly, it represents an infinite number of differential equations, one for each Fourier mode of each operator. In general, these Fourier modes are quite complicated; even if $G$ depends only on the conformal time $\eta, \eta$ is expandable in Fourier modes and the metric is generally a nontrivial function. Finally, it is difficult to understand and interpret the wave functional in spacetime since the spacetime time coordinate of the string depends on the worldsheet spatial coordinate, as it does in Equation (23). With this in mind we will use only the $\sigma$-independent modes of Equation (29) (in other words, the $L_{0}$ and $\bar{L}_{0}$ constraints), and we will set $\eta=\eta(\tau)$.

If we write the Fourier expansion of our string modes as

$$
X^{\mu}(\tau, \sigma)=\sum_{n=-\infty}^{\infty} X_{n}(\tau) e^{i n \sigma}
$$


and use the reality constraints, we find that $X_{n}=X_{-n}^{*}$. Let

$$
X_{n}^{\mu}=X_{n}^{1 \mu}+i X_{n}^{2 \mu},
$$

so that $X_{-n}=X_{n}^{1}-i X_{n}^{2}$. We then find that the $L_{0}$ part of (29) is, for the homogeneous spacetimes we are interested in,

$$
\begin{gathered}
\left\{\frac{1}{\sqrt{G(\eta)}} \frac{\partial}{\partial \eta} \sqrt{G(\eta)} G^{\eta \eta} \frac{\partial}{\partial \eta}-\sum_{k=1}^{d-1} \sum_{n=1}^{\infty} G^{k k}(\eta)\left(\left(\nabla_{k, n}^{(1)}\right)^{2}+\left(\nabla_{k, n}^{(2)}\right)^{2}\right)-\right. \\
\sum_{k=1}^{d-1} G^{k k}(\eta)\left(\frac{\partial}{\partial X_{0}^{k}}\right)^{2}+ \\
\left.\sum_{k=1}^{d-1} \sum_{n=1}^{\infty} n^{2}\left(\left(X_{1, n}^{k}\right)^{2}+\left(X_{1, n}^{k}\right)^{2}\right)^{2}-\xi \mathcal{R}\right\} \Psi\left[\eta,\left\{X_{n}\right\}\right]=0 \\
\left\{\sum_{k=1}^{d-1} \sum_{n=1}^{\infty} n\left(X_{1, n}^{k} \frac{\partial}{\partial X_{2, n}^{k}}-X_{2, n}^{k} \frac{\partial}{\partial X_{1, n}^{k}}\right)\right\} \Psi\left[\eta,\left\{X_{n}\right\}\right]=0
\end{gathered}
$$

(where we take the metric to be diagonal). Here $\nabla_{\mu, n}^{(i)}=\partial / \partial X_{n}^{(i) \mu}$. Imposing the final constraint in Equation (32) becomes much simpler if we set

$$
X_{n}=R_{n} e^{i \phi_{n}},
$$

as Equation (32) becomes:

$$
\begin{aligned}
& \left\{\frac{1}{\sqrt{G}} \frac{\partial}{\partial \eta} \sqrt{G} G^{\eta \eta} \frac{\partial}{\partial \eta}-\right. \\
& \quad \sum_{k=1}^{d-1} \sum_{n=1}^{\infty} G^{k k}\left(\frac{1}{R_{n}^{k}} \frac{\partial}{\partial R_{n}^{k}} R_{n}^{k} \frac{\partial}{\partial R_{n}^{k}}+\frac{1}{\left(R_{n}^{k}\right)^{2}}\left(\frac{\partial}{\partial \phi_{n}^{k}}\right)^{2}\right)- \\
& \left.\quad \sum_{k=1}^{d-1} \sum_{n=1}^{\infty} n^{2}\left(R_{n}^{k}\right)^{2}-\xi \mathcal{R}\right\} \Psi\left[\eta,\left\{X_{n}\right\}\right]=0 \\
& \sum_{k=1}^{d-1} \sum_{n=1}^{\infty} n \frac{\partial}{\partial \phi_{n}^{k}} \Psi\left[\eta,\left\{R_{n}, \phi_{n}\right\}\right]=0 .
\end{aligned}
$$

Note in particular that these constraints commute with $\partial / \partial \phi_{n}^{k}$ for all $n, k$, so that we may let

$$
\Psi[\{R, \phi\}]=\exp \left\{i \sum_{k, n} l_{k, n} \phi_{n}^{k}\right\} \Psi[\{R\}],
$$

and thus make the substitution

$$
\frac{\partial}{\partial \phi_{n}^{k}} \longrightarrow i l_{k, n}
$$


It will be useful at this point to write down the stationary states of (34) in flat space. We will add a term $\mathcal{E}_{0}^{2} \Psi$ to this equation in order to explicitly subtract off the zero-point energy: this will include the usual tachyon shift $\left(-m_{0}^{2}\right)$ if we are working with the bosonic string. In flat space the equation clearly separates into an infinite number of 2-dimensional harmonic oscillators, one for each $(k, n)$, with the single constraint that

$$
\sum_{k=1}^{d-1} \sum_{n=1}^{\infty} n l_{k, n}=0
$$

We also get a Klein-Gordon equation for the $X_{0}$ wave functions. The final wave function may then be written in terms of associated Laguerre polynomials. If we leave the scale factor $\Omega$ of the metric in explicitly, we find that

$$
\begin{aligned}
\Psi_{\left\{m_{k, n}, l_{k, n}\right\}}= & e^{-i E \eta+i \vec{q} \cdot \vec{X}_{0}} \times \\
& \prod_{k, n_{k}} e^{-n_{k} \Omega\left(R_{n}^{k}\right)^{2} / 2}\left(n \Omega\left(R_{n}^{k}\right)^{2}\right)^{l_{k, n} / 2} L_{m_{k, n}}^{l_{k, n}}\left(n \Omega\left(R_{n}^{k}\right)^{2}\right) e^{i l_{n, k} \phi_{n}^{k}} .
\end{aligned}
$$

Here

$$
\begin{aligned}
E^{2} & =\vec{q}^{2}+\sum_{n, k} 4 n \Omega\left(m_{k, n}+\frac{l_{k, n}+1}{2}\right)-\mathcal{E}_{0}^{2} \\
& =\sum_{n, k} 4 n \Omega\left(m_{k, n}+\frac{1}{2}\right)-\mathcal{E}_{0}^{2}
\end{aligned}
$$

and the final line in (39) comes from (37). Note that $\Omega$ acts as the frequency of the harmonic oscillator, and the only difference between this system and a product of nonrelativistic harmonic oscillators is that $E$ gets replaced by $E^{2}$. As usual, $\mathcal{E}_{0}$ is defined such that

$$
E^{2}=\vec{q}^{2}+\sum_{n, k} 4 n m_{k, n}-m_{0}^{2} .
$$

\subsection{Approximations for cosmological models}

Let

$$
d s^{2}=-d t^{2}+\sum_{k=1}^{d-1} a_{k}(t) d x_{k}^{2} .
$$

Putting this metric into Equation (34), we find that the scale factors $a(t)$ act as time-dependent masses for the different 2D harmonic oscillators. In this case, Equation (34) is not separable, and no interesting scale factors yielding 
exact solutions are known to us. (This is in contrast to the non-relativistic harmonic oscillator with a time-dependent mass and/or frequency, where one can reduce the quantum problem to the classical problem by introducing a Gaussian wave packet - essentially a coherent-state wavepacket - with timedependent coefficients (Peremolov and Popov 1969). There is no solution in the relativistic case as there are no analogs of coherent states: even with a time-independent mass and frequency, the relativistic dispersion relation causes the wave packet to spread.) We will instead examine Equation (34) in certain limiting cases.

\subsubsection{The adiabatic limit}

The simplest limit to study is the limit of an adiabatically changing universe, where $a_{k}=a_{k}(\rho t), 1 / \rho \ll \ell_{s t r}$ (note we have so far set $\ell_{s t r}=1$ ). Let us work to lowest adiabatic order; since the curvature scalar is second order in derivatives of the metric, and thus $\mathcal{O}\left(\rho^{2}\right)$, we will ignore this term for the present. To this order, the oscillator number should be an adiabatic invariant, so we use the following variant of the flat space energy eigenfunctions:

$$
\begin{aligned}
& \Psi_{m_{k, n}, l_{k, n}}^{\text {ad. }}[\{R, \phi\}]= \\
& \quad \prod_{k, n_{k}} e^{-n_{k} a_{k}(\eta)\left(R_{n}^{k}\right)^{2} / 2}\left(n a_{k}(\eta)\left(R_{n}^{k}\right)^{2}\right)^{l_{k, n} / 2} L_{m_{k, n}}^{l_{k, n}}\left(n a_{k}(\eta)\left(R_{n}^{k}\right)^{2}\right) e^{i l_{k, n} \phi_{n}^{k}} .
\end{aligned}
$$

If we now expand the total wave function in this basis using time dependent coefficients,

$$
\Psi=\sum_{\left\{m_{k, n}, l_{k, n}\right\}} c_{\left\{m_{k, n}, l_{k, n}\right\}}(t) \Psi_{\left\{m_{k, n}, l_{k, n}\right\}}^{\mathrm{ad}},
$$

then applying Equation (34) to this wave function will give us a coupled set

of differential equations for the coefficients $c_{\left\{m_{k, n}, l_{k, n}\right\}}(t)$, subject to the initial conditions appropriate to the question one is asking.

We would like to compute the probability of particle production. As usual, we first need to define the positive frequency modes of the string field in the far past and future. The standard definition is the definition of adiabatic positive frequency modes (see Birrell and Davies (1982) for an explanation and references): we ask that the modes we define as "positive frequency" be those that match exactly onto positive frequency WKB solutions in the infinite past,

$$
\Psi_{\left\{m_{k, n}, l_{k, n}\right\}}^{\mathrm{ad}} \frac{1}{\sqrt{E(t)}} \exp \left\{-i \int_{-\infty}^{t} d t^{\prime} E\left(t^{\prime}\right)\right\},
$$

where for our spacetime

$$
E(\eta)=\sqrt{\sum_{k} \frac{\left(q^{k}\right)^{2}}{a_{k}(t)}+\sum_{n} 4 n m_{k, n}+\xi \mathcal{R}} .
$$


Ideally, we would find the appropriate exact wave function that matches onto this WKB solution and look for the negative frequency components in the usual manner: in lieu of this, we would like a WKB approximation for the wave function at all times. Two problems arise, however. The first problem is that the negative frequency components will not be present at any order in the perturbation series one would get from plugging (43) into (34). The reason is identical to the reason one does not find above-barrier reflection in semiclassical non-relativistic quantum mechanics to any perturbative order in $\hbar$. The second problem is that the terms in the equations for the coefficients $c_{m, l}(t)$ in Equation (43) which are higher order in $\rho$ come from applying the time derivatives to $\Psi^{\text {ad. }}$, which depend implicitly on time through $a_{k}$. The reader may easily verify that these terms are also multiplied by factors of $m_{k, n}$ and higher powers of $m$, because of the recursion relations for Laguerre polynomials. Thus if $m_{k, l} \rho \gg 1$, the adiabatic approximation breaks down. We will treat this case below.

In the case that the adiabatic approximation holds, there is still a trick for extracting the negative frequency part of the wave function in the far future. Audretsch (1979) has pointed out, for scalar particles in curved spacetime, that the particle production calculation is almost identical to the calculation of abovebarrier reflection in nonrelativistic quantum mechanics, since one can generally (for homogeneous spacetimes) separate the time and space parts of the wave equation and write the former as

$$
\frac{\partial^{2}}{\partial t^{2}} f+\omega(t)^{2} f=0
$$

The difference is that in above-barrier reflection, we want the incident component of the wave function to be normalized to unity and the transmitted wave function to be purely outgoing. Then, if the amplitude of the transmitted wave is $T$ and the amplitude of the reflected wave is $R$ (assuming that only one value of $\left|\omega_{t=\infty}\right|$ contributes to the final wave function, though the discussion may be easily generalized) probability conservation requires that

$$
|T|^{2}+|R|^{2}=1
$$

On the other hand, in the case of non-static spacetimes, we wish the wave function in the far past to be purely positive frequency. If this incoming wave function is normalized to unity, and the coefficients of the positive and negative frequency components of the outgoing wave function are denoted $\alpha$ and $\beta$ (again we assume that only one magnitude of the frequency contributes to the final wave function), probability conservation requires that

$$
1+|\beta|^{2}=|\alpha|^{2}
$$

If we write $\omega=\sqrt{2(E-V(t))}$ the analogy is obvious. In general, $\omega$ will vanish in the upper half complex $t$-plane at some point (or set of points) $t_{0}$, and also 
at $t_{0}^{*}$. The square of the reflection coefficient in the WKB approximation has been derived by Pokrovskii and Khalatnikov (1961):

$$
|R|^{2}=\exp \left\{-2 \operatorname{Im} \int_{t_{0}^{*}}^{t_{0}} d t \omega(t)\right\} .
$$

In our case, if we substitute $E$ for $\omega,|R|^{2}$ becomes the square of the Bogolubov coefficient multiplying the negative frequency WKB solution in the far future (Audretsch 1979).

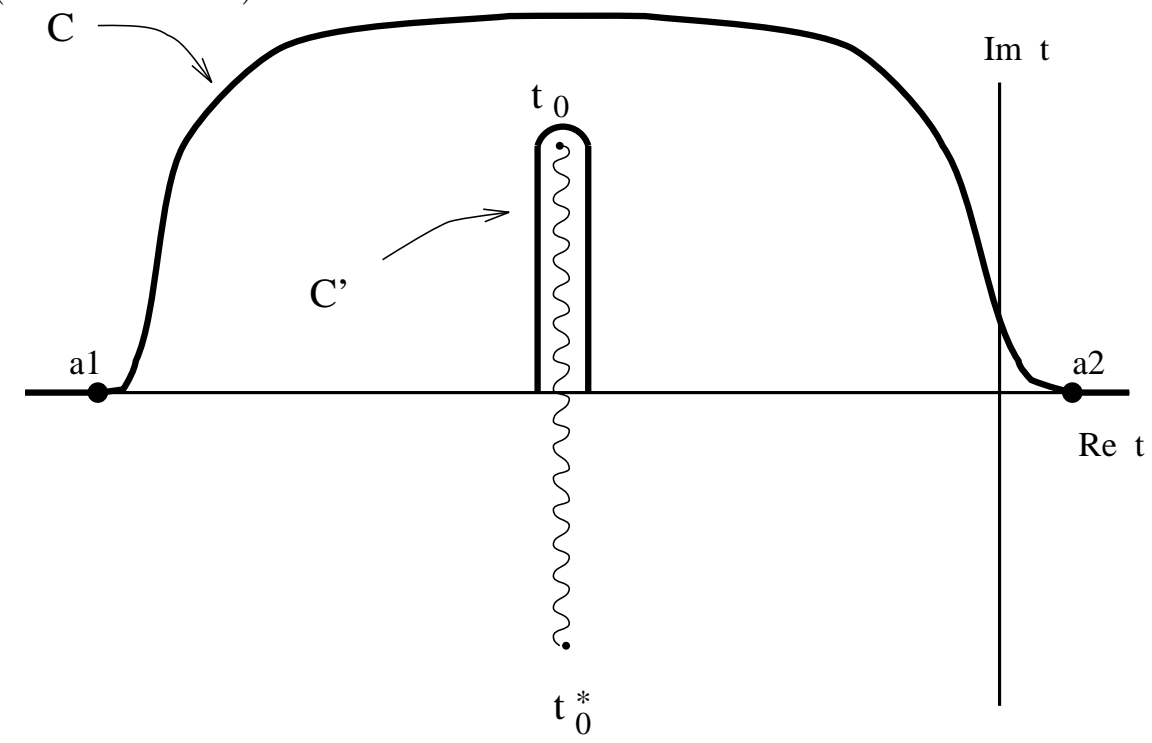

Figure 1

Complex time paths. $t_{0}$ and $t_{0}^{*}$ are the classical turning points of the system. Curve $C$ denotes a path which moves around the square root branch point, while staying far enough away for the adiabatic or semiclassical approximation to hold. For the purposes of computing the production amplitude the integral $\int d t E$ may be performed along curve $C^{\prime}$.

Before we continue, let us describe a more heuristic derivation of Equation (49) (see also Landau and Lifshitz (1977) and Migdal (1977), from which this discussion was taken). As we have seen, the time dependence of our system has the form of Equation (44). The expression for $E$ in equation (45) contains square root branch cuts in the complex $t$ plane (see Figure 1). In order to detect the part of the wave function with negative energy, we should go around the end of the branch cut in $E$. If we stay far enough away from this endpoint, then the adiabatic approximation will remain valid. (The approximation will break down near the turning point, where $E=0$, since one requires for the approximation that the time variation of the wave function be more rapid than 
the time variation of the background.) Along the contour, labeled $C$ in Figure 1, the integral $\int d t E$ will pick up an imaginary component, which will give us the real part of the Bogolubov coefficient $\beta$. This real part will not change if we move the points $a 1, a 2$, where the contour $C$ leaves the real axis, adjacent each side of the branch cut; so long as $C$ does not enclose any singularities other than the branch cut, we may then shrink the contour down to the curve $C^{\prime}$ which surrounds the branch cut as shown. A little manipulation of the integral should convince the reader that the imaginary part of the integral in Equation (49) is then the correct result for the real part of the phase picked up when traveling on contour $C$. Since $\exp \{-E / \rho\}$ will be small in the adiabatic limit, the correction for the correct normalization of the wave function will be even smaller, and we may identify the expression shown in equation (49) with $|\beta|^{2}$. This discussion can be easily generalized to systems with several turning points in the complex plane (Pokrovskii and Khalatnikov 1961, Landau and Lifshitz 1977, Migdal 1977, Audretsch 1979).

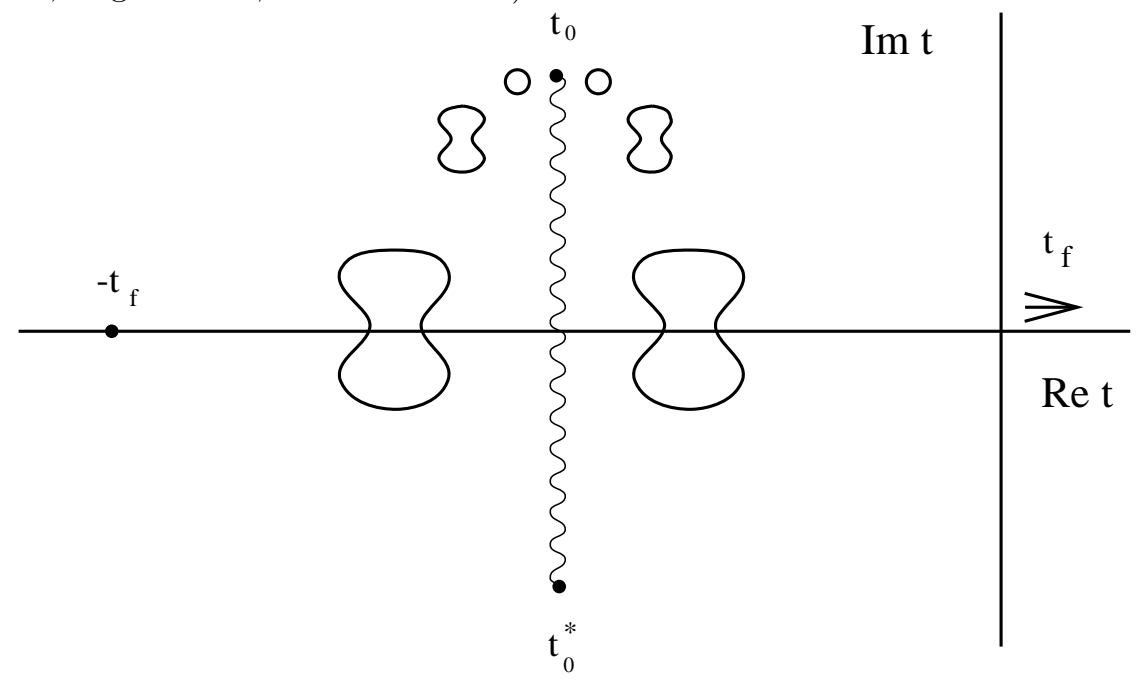

Figure 2

Pair production of strings. Here we depict the configuration of the classical string as it passes around the branch point in the complex $t$ plane. One may interpret this as a pair production process beginning at $t_{0}$; the string which reaches the real axis on the left of the branch cut is positive frequency and the string which reaches the right side of the branch cut is negative frequency.

One can envision the pair production process via the classical trajectory of the string on $C^{\prime}$. Since the energy is small near the turning point, the size of the string will be small as well. The classical trajectory of the string describes two zero-size, zero-energy strings being produced at $t_{0}$, and growing as they travel in imaginary time along opposite sides of the branch cut (see Figure 2). 


\subsubsection{The semiclassical limit}

In the limit that the occupation numbers $m_{k, n}$ are large, the adiabatic approximation breaks down and the semiclassical approximation is appropriate. Let us write the wave function as

$$
\Psi=\sum_{j} D_{j}\left(t,\left\{X_{n}\right\}\right) e^{\frac{i}{\alpha^{\prime}} S_{j}\left(t,\left\{x_{n}\right\}\right)} .
$$

In the semiclassical limit, where the frequencies (excitation numbers and center of mass momentum) are large, $S$ will solve the Hamilton-Jacobi equation

$$
\begin{aligned}
-\left(\frac{\partial S}{\partial t}\right)^{2} & +\sum_{k} \frac{\left(q^{k}\right)^{2}}{a_{k}}+\sum_{k, n} \frac{1}{a_{k}(t)}\left[\left(\frac{\partial S}{\partial R_{n}^{k}}\right)^{2}+\left(\frac{1}{R_{k}^{n}} \frac{\partial S}{\partial \phi_{n}^{k}}\right)^{2}\right]+a_{k}(t)\left(R_{n}^{k}\right)^{2} \\
& +\xi \mathcal{R}-m_{0}^{2}=0
\end{aligned}
$$

where $S$ defines the momentum as a function of position via $p_{n}=\partial S / \partial q_{n}, p$ is a multivalued function defined by a curve $\mathcal{C}$ in phase space (see Figure 3 ) and the sum in $(50)$ is the sum over the branches of the function $p(q)$ defined by $\mathcal{C}$. If we foliate phase space with curves $\mathcal{C}=\mathcal{C}(\mathcal{P})$, then we may write $D_{j}$ as the square root of the Van Vleck determinant

$$
D_{j}=\text { constant } \times \sqrt{\left|\frac{\partial^{2} S_{j}}{\partial q_{l} \partial \mathcal{P}_{k}}\right|} .
$$

(This geometric picture is taken from Berry and Balasz (1979) and is due to Maslov (1965). See Berry (1981) for further review and references.) In general the condition for the semiclassical approximation to hold is that:

$$
\begin{aligned}
& \frac{\partial^{2} S}{\partial q_{n}^{2}} \ll\left(\frac{\partial S}{\partial q_{n}}\right)^{2} \\
& \frac{\partial^{2} S}{\partial t^{2}} \ll\left(\frac{\partial S}{\partial t}\right)^{2} .
\end{aligned}
$$

This is the usual criteria for the validity of the WKB approximation. 

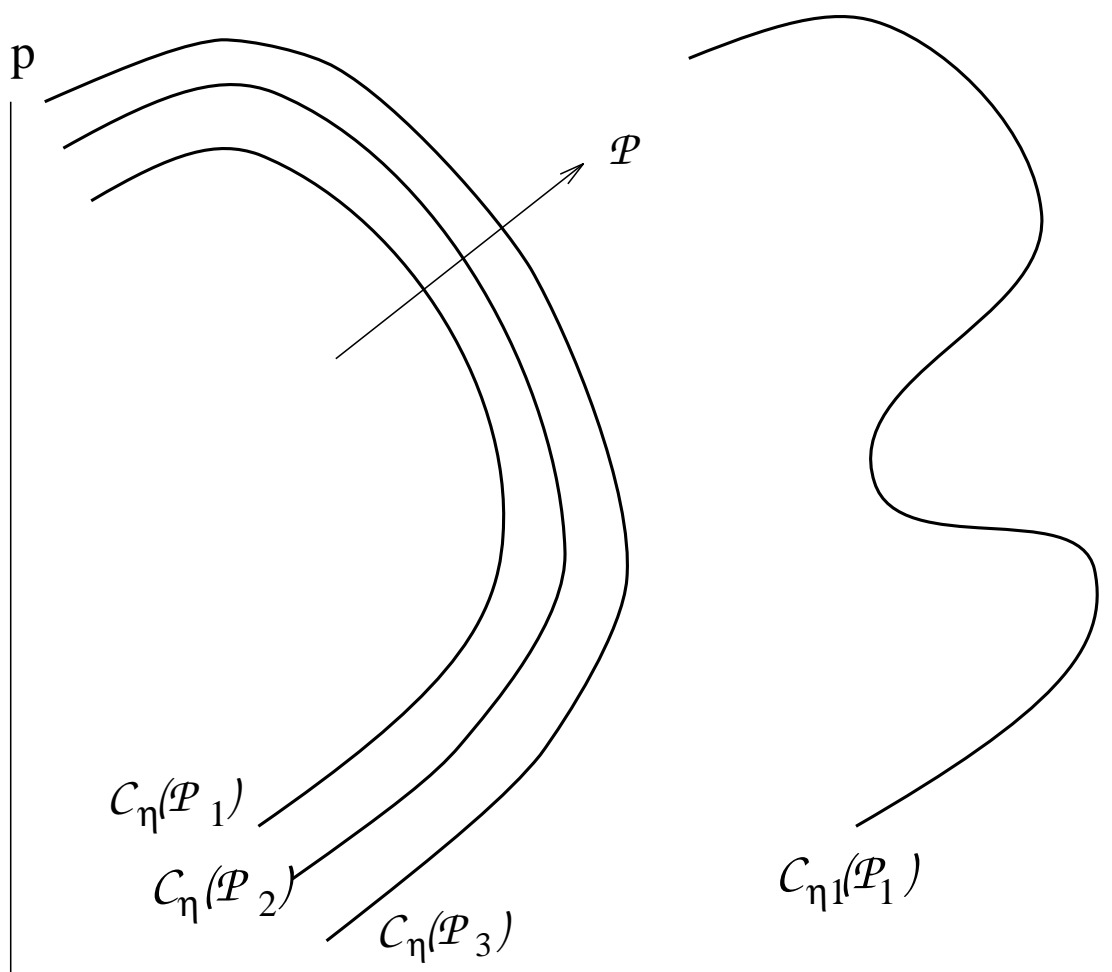

q

Figure 3

Curves $p(q)$ in phase space. The $n$-dimensional surface $\mathcal{C}_{\eta}(\mathcal{P})$ defines a multivalued function $p_{k}\left(\left\{q_{l}\right\}\right)$ at time $\eta$ in a $2 n$-dimensional phase space. $\mathcal{P}$ labels a family of n-dimensional curves which foliate phase space. $\mathcal{C}_{\eta 1}\left(\mathcal{P}_{1}\right)$ is found by evolving each point on $\mathcal{C}_{\eta}\left(\mathcal{P}_{1}\right)$ from time $\eta$ to time $\eta^{\prime}$ via Hamilton's equations.

Note that in general the Hamilton-Jacobi equation for a relativistic system should look like

$$
\frac{\partial S}{\partial \tau}+\mathcal{H}\left(t, x, E=\frac{\partial S}{\partial t}, p=\frac{\partial S}{\partial x}, \tau\right)=0,
$$

if we are to treat space and time on an equal footing. $\tau$ should be an arbitrary parameter describing the world line of this system, i.e. the $\tau$ dependence of the system should be trivial. Thus, we arrive at Equation (51), where

$$
\begin{aligned}
& \mathcal{H}\left(t, E, R_{n}^{k}, p_{R, n}^{k}, \phi_{n}^{k}, p_{\phi, n}^{k}\right)= \\
& \quad-E^{2}+\sum_{k} \frac{\left(q^{k}\right)^{2}}{a_{k}}+\sum_{k, n}\left\{\frac{\left(p_{R, n}^{k}\right)^{2}}{a_{k}(t)}+\frac{\left(p_{\phi, n}^{k}\right)^{2}}{\left(R_{n}^{k}\right)^{2} a_{k}(t)}+a_{k}(t)\left(R_{n}^{k}\right)^{2}\right\}
\end{aligned}
$$




$$
+\xi \mathcal{R}-m_{0}^{2}=0 .
$$

The simplest way forward is to simply solve for the constraints, by letting $E$, the generator of $t$ translations, become the Hamiltonian and using Equation (56) to write

$$
\begin{aligned}
E\left(R, p_{R}, \phi, p_{\phi}, t\right) & =\sqrt{\sum_{k} \frac{\left(q^{k}\right)^{2}}{a_{k}}+\sum_{k, n}\left\{\frac{\left(p_{R, n}^{k}\right)^{2}}{a_{k}(t)}+\frac{\left(p_{\phi, n}^{k}\right)^{2}}{\left(R_{n}^{k}\right)^{2} a_{k}(t)}+a_{k}(t)\left(R_{n}^{k}\right)^{2}\right\}+\left(\xi \mathcal{R}-m_{0}^{2}\right.}=0 .
\end{aligned}
$$

This also comes from putting the term $(\partial S / \partial t)^{2}$ on the right hand side of Equation (51) and taking the square root of the equation.

Now we must solve the Hamilton-Jacobi equation

$$
\frac{\partial S}{\partial t}+E=0 .
$$

We describe the solution as follows (this discussion is patterned after that in Berry and Balazs (1979), though our representation for $S$ is different). At some initial time, say $t=0$, we start with a curve $\mathcal{C}_{0}$ in phase space which defines the desired initial wave function via (50). This curve will evolve forward in time, as one can see by taking each point on the curve as an initial condition for Hamilton's equations, to a curve $\mathcal{C}_{t}$ at time $t$. This will define a family of functions $p(q, t)$. Assuming no new caustics in $p(q, t)$ develop, the solution to the Hamilton-Jacobi equation can be written, to within a constant, as:

$$
S=\int_{q_{n, 0}}^{q_{n}} p_{n}\left(q, t_{0}\right) d q_{n}-\int_{t_{0}}^{t} E\left(q_{n}, p_{n}\left(q_{n}, t^{\prime}\right), t^{\prime}\right) d t^{\prime} .
$$

Here the $q_{(n, 0)}$ are arbitrary constants. A straightforward application of Hamilton's equations confirms that

$$
\frac{\partial S}{\partial q}=p(q),
$$

and that $S$ as defined in Equation (59) satisfies the Hamilton-Jacobi equations.

One may compute string production in the same fashion as one would in the adiabatic approximation: here one would search for turning points in the complex time plane for $p_{n}$ and $E$. To see this procedure in an explicit model we turn to the next section.

\section{$5 \quad$ An isotropically expanding model}

We would now like to examine a model from which we may extract a few results. For the reasons laid out in the beginning of the previous section, we would like to 
examine a model in which the far past and far future correspond to Minkowski space and there is a finite period of expansion near $\eta=0$. At present the one case we have found to be tractable is the case of isotropic expansion. In this case we will work in conformal time:

$$
d s^{2}=C(\eta)\left(-d \eta^{2}+\sum_{k}\left(d x^{k}\right)^{2}\right) .
$$

A simple scale factor with the desired properties is $C(\eta)=A+B \tanh \rho \eta$, where $A-B=1$ and $A+B=\Omega$, the final scale factor. The conformally coupled KleinGordon equation in a two-dimensional spacetime with this scale factor has been solved exactly and examined by Bernard and Duncan (1977); the time part of the equation is identical to the Schrödinger equation in a special case of the Eckhardt-Sauter potential (Eckhardt 1930, Sauter 1932). It will be useful as a warmup exercise to compute the production probability for particles in this spacetime using the WKB approximation, since we have an exact solution to compare it to. After some work we will find the semiclassical result for string production to be nearly identical.

\subsection{Review of the Klein-Gordon equation}

For a particle with mass $m$, we are looking for solutions which look in the far past and far future like:

$$
\begin{aligned}
t \longrightarrow-\infty: & \phi(x, t) & \sim e^{-i \omega_{-} t+i q x} \\
t \longrightarrow \infty: & \phi(x, t) & \sim \alpha_{q} e^{-i \omega_{+} t+i q x}+\beta_{q} e^{i \omega_{+} t+i q x},
\end{aligned}
$$

where

$$
\omega_{ \pm}^{2}=q^{2}+(A \pm B) m^{2} .
$$

Bernard and Duncan (1977) found exact solutions to the Klein-Gordon equation (in the form of hypergeometric functions) which match to (62). The exact expression for the production probability is

$$
\left|\beta_{k}\right|^{2}=\frac{\sinh ^{2}\left(\pi\left(\omega_{+}-\omega_{-}\right) / 2 \rho\right)}{\left.\sinh \left(\pi \omega_{-} / \rho\right) \sinh \left(\pi \omega_{+} / \rho\right)\right)} .
$$

In the limit of small $\rho$ (or large energies and energy differences), this expression becomes

$$
\left|\beta_{k}\right|^{2}=e^{-2 \pi \omega_{-} / \rho}
$$

Using the WKB approximation, we find that Equation (49) becomes

$$
\left|\beta_{k}\right|^{2}=\exp \left\{-2 \operatorname{Im} \int_{\eta_{0}^{*}}^{\eta_{0}} \sqrt{k^{2}+(A+B \tanh \rho t) m^{2}}\right\},
$$


where

$$
\eta_{0}=\frac{i \pi}{2 \rho}+\frac{1}{2 \rho} \ln \left(\frac{\omega_{-}}{\omega_{+}}\right) .
$$

If we make the substitution

$$
\eta=\ln \left(\frac{\omega_{-}}{\omega_{+}}\right)+\frac{\ln z}{\rho},
$$

then the integral in Equation (66) becomes

$$
\frac{\omega_{-}}{2 \rho} \oint_{|z|=1} \frac{d z}{z} \sqrt{\frac{z+1}{z+\frac{\omega_{+}}{\omega_{-}}}}=\frac{i \pi \omega_{-}}{\rho} .
$$

Plugging this into Equation (66) we find that this approximation reproduces Equation (65).

\subsection{Strings in an expanding universe}

For states for which the adiabatic approximation holds, it is easy to see that if we ignore $\left(\xi \mathcal{R}-m_{0}^{2}\right)$ in the equation, we may use the approximations described to arrive at Equation (65). However, states for which this approximation holds are not generic at sufficiently high mass levels. String theory contains an infinite number of states with a multiplicity growing as the mass. For a given level $M=$ $(\text { mass })^{2}$ of string excitation, states corresponding to high Fourier components $K$ of the string excited to low oscillator levels $m_{K}$, such that $m_{K} \rho \ll 1$, can still be treated with the adiabatic method. But in general the typical size of the string will be large, reflecting a large number of Fourier components excited to a high oscillator level. We will find that while some distributions of the energy for a given $M$ are intractable even in the semiclassical limit, i.e. those with a few Fourier components highly excited, a configuration with $N \gg 1$ Fourier modes excited may be treated in a $1 / N$ expansion. The latter type of configuration should be fairly generic for sufficiently large mass; we expect that most configurations at a given total oscillator level $n$ will have their energy partitioned among many oscillator modes.

In conformal coordinates, the scale factor appears as a time-dependent frequency rather than a time-dependent mass; thus, the energy (the generator of $\eta$ translations) is

$$
E=\sqrt{\vec{q}^{2}+C(\eta)\left(\xi \mathcal{R}-m_{0}^{2}\right)+\sum_{k, n}\left(p_{R, n}^{k}\right)^{2}+\left(n C(\eta) R_{n}^{k}\right)^{2}+\left(\frac{p_{\phi, n}^{k}}{R_{n}^{k}}\right)^{2}} .
$$

Hamilton's equations are:

$$
\dot{R}_{n}^{k}=\frac{p_{R, n}^{k}}{E}
$$




$$
\begin{aligned}
& \dot{p}_{R, n}^{k}=\left(\frac{\left(p_{\phi, n}^{k}\right)^{2}}{\left(R_{n}^{k}\right)^{2}}-(n C(\eta))^{2} R_{n}^{k}\right) / E \\
& \dot{\phi}_{n}^{k}=\frac{p_{\phi, n}^{k}}{E\left(R_{n}^{k}\right)^{2}} \\
& \dot{p}_{\phi, n}^{k}=0 .
\end{aligned}
$$

(There is also the remaining constraint $\sum_{k, n} n l_{k, n}=0$.) These equations may be converted into equations of motion for $R$ and $\phi$ :

$$
\begin{aligned}
& \ddot{R_{n}^{k}}+\left(\frac{n C(\eta)}{E}\right)^{2} R_{n}^{k}-\frac{\left(p_{\phi, n}^{k}\right)^{2}}{E^{2}\left(R_{n}^{k}\right)^{3}}+\frac{\dot{E}}{E} \dot{R_{n}^{k}}=0 \\
& \ddot{\phi_{n}^{k}}+\frac{2 p_{\phi, n}^{k} \dot{R_{n}^{k}}}{\left(R_{n}^{k}\right)^{3}}+\frac{\dot{E}}{E} \dot{\phi_{n}^{k}}=0 .
\end{aligned}
$$

Note that without the $\dot{E} / E$ terms, Equation $(72)$ looks like the equations for a harmonic oscillator with frequency

$$
\bar{\omega}_{n}=\frac{n C(\eta)}{E} .
$$

If a single oscillator is excited, Equation (72) becomes quite difficult to solve in the limit $E \rho / \omega \gg 1$. The $\dot{E} / E$ term is of lower adiabatic order, but the reduced frequency is smaller still. The period of oscillation in spacetime time is much longer than the time scale $\rho^{-1}$. Whether $\omega R / E$ is of order 1 , of order $\rho$, or of order $1 / E$, and thus whether the velocity- or position-dependent force dominates, will depend on where in the potential the oscillator term is. At present, we have no ideas to offer for a solution in this case.

However, as we have stated above, most of the states at a given large mass will be in oscillators with many Fourier modes excited - call this number $N$. We will attempt to solve for the classical equations to lowest order in $1 / N$. This is similar to the expansion in the size of the string we presented two sections ago; the string in such a state will be large and slow. We will assume that for a given oscillator mode $(k, n)$ with energy $E_{k, n}, E_{k, n} \rho / \omega \gg 1$ and $N \gg 1$; thus $E \gg E_{k, n}$ for any $(k, n)$.

Using Hamilton's equations we can see that

$$
\frac{\dot{E}}{E}=\frac{\dot{C}}{C} \frac{\sum_{k, n} n^{2} C^{2}\left(R_{n}^{k}\right)^{2}}{E^{2}} .
$$

The expression multiplying $\dot{C} / C$ is $\mathcal{O}(1)$ both in $1 / N$ and in $\rho$. The positiondependent terms in (72) are of order $1 / N$. Even though $N \rho \gg 1$, the oscillator terms will dominate for most of the life of the universe, where the $\tanh (\rho \eta)$ term in $C$ is exponentially suppressed and so not changing much. More specifically, 
note that

$$
\begin{aligned}
\frac{\dot{C}}{C} & =\frac{B \rho \operatorname{sech}^{2} \rho \eta}{A+B \tanh \rho \eta} \\
& \sim B \rho e^{-2 \rho|\eta|} \text { for } \eta \longrightarrow \pm \infty .
\end{aligned}
$$

Thus, noting that Hamilton's equations tell us that $\dot{R} \sim \mathcal{O}(1 / \sqrt{N})$, so long as

$$
|\eta|>\frac{1}{2 \rho} \ln \{|B| \rho \sqrt{N}\}=\eta_{f},
$$

the position dependent forces dominate and the system behaves as a collection of simple harmonic oscillators. Since

$$
\dot{E} \sim \frac{\dot{\omega}}{\omega} \mathcal{O}(\sqrt{N}),
$$

for $|\eta|>\eta_{f}$ the change in $E$ (or in $\omega$ ) becomes very small exponentially rapidly, and the system behaves as set of oscillators with frequency

$$
\bar{\omega}_{ \pm}=\frac{n C( \pm \infty)}{E( \pm \infty)} .
$$

Note that the factor of $1 / E$ means that the potential is quite shallow and in general each Fourier mode will have a spatial extent of order $\sqrt{N}$ : the string will be quite large, as promised. For $|\eta|<\eta_{f}$, the velocity dependent term dominates. Keeping only these terms and the second derivative in (72), we find that

$$
\begin{aligned}
\dot{R_{n}^{k}} & =\frac{\alpha_{R, n}^{k}}{E} \\
\dot{\phi}_{n}^{k} & =\frac{\alpha_{\phi, n}^{k}}{E},
\end{aligned}
$$

where the $\alpha$ are integration constants and will be fixed by matching this solution to the $|\eta|>\eta_{f}$ region. Thus the velocities are $\mathcal{O}(1 / \sqrt{N})$ : we shall call this region the "frozen region" (thus the suffix in $\eta_{f}$ ). The changes in these coordinates are:

$$
\delta(R, \phi) \sim 2 \eta_{f}(\dot{R}, \dot{\phi}) \sim \frac{1}{\rho \sqrt{N}} \ln \{|B| \rho \sqrt{N}\} .
$$

As long as $\rho \sqrt{N} \gg 1$, the change in the coordinate positions is small; therefore, we will approximate the string in this region as being fixed in comoving coordinates, so that its proper size grows with the scale factor, as we saw two sections ago.

Physically, the picture that we have is that strings which are larger than a horizon radius during expansion become "frozen" and expand with the scale factor without oscillating, as different parts of the string are no longer in causal contact with each other. The entire history of the string can be divided into three regions: 
- $-\infty<\eta<\eta_{f}$. Strings oscillate freely and energy is conserved. Here we may expand the string wave function in stationary states of the initial Hamiltonian.

- $-\eta_{f}<\eta<\eta_{f}$ (the "frozen" region.) Classically, strings larger than the horizon size do not oscillate coherently since different regions of the string are causally disconnected from each other. Instead, the string grows with the scale factor. Equivalently, in conformal coordinates the string wave function is frozen as the harmonic oscillator potentials decrease in width.

- $\eta_{f}<\eta<\infty$. The wave function at $\eta=\eta_{f}$ is no longer a stationary state of the final Hamiltonian: we may, within our approximation, decompose it into eigenstates of the final Hamiltonian, just as in the sudden approximation.

In the language of Berry and Balazs (1979), if we are looking for a wave function corresponding to an energy eigenstate with energy $E_{-}$in the far past, we wish to start with a curve $\mathcal{C}_{-\infty}$ in phase space corresponding to the torus defined by the equation $E=E_{-}$in phase space. In this region the energy of each oscillator, $E_{k, n}$, is a constant of motion, so that

$$
p_{R, n}^{k}= \pm \sqrt{\left(E_{n}^{k}\right)^{2}-\bar{\omega}_{-}^{2}\left(R_{n}^{k}\right)^{2}-\left(\frac{l_{n}^{k}}{R_{n}^{k}}\right)^{2}} .
$$

The branch of the square root changes when we reach a caustic in $p(q)$ and, as stated, we must sum $\exp \{i S\}$ over the branches of $p$. In our approximation, when $\eta<-\eta_{f}$ the energy is effectively time-independent and the resulting semiclassical wave function is just the semiclassical approximation to the product of harmonic oscillator wave functions. In the "frozen" region, since $\dot{R}, \dot{\phi}$, and $\dot{p}_{R}$ are $\mathcal{O}(1 / \sqrt{N})$, to lowest order in our $1 / \sqrt{N}$ expansion the curve $\mathcal{C}_{\eta}$ will remain frozen. Within our approximation we may write the classical action as:

$$
\begin{aligned}
S\left(R_{n}^{k}, \phi_{n}^{k}, \eta\right) & =\sum_{k, n}\left\{l_{n}^{k} \phi_{n}^{k}+\int_{R_{n, 0}^{k}}^{R_{n}^{k}} p_{R, n}^{k}\left(R_{n}^{k}, \eta_{0}\right)\right\} \\
-\int_{-\infty}^{\eta} d \eta^{\prime} & \sqrt{\sum_{k, n}\left\{\left(E_{n}^{k}\right)^{2}+\left(n C\left(\eta^{\prime}\right)^{2}-n^{2}\right)\left(R_{n}^{k}\right)^{2}\right\}+C\left(\eta^{\prime}\right)\left(\xi \mathcal{R}\left(\eta^{\prime}\right)-m_{0}^{2}\right)} .
\end{aligned}
$$

The curvature term in the expression for $E$ is $\mathcal{O}(1 / N)$ smaller than the other terms, so we shall ignore it. As we leave the frozen region, we may calculate the negative frequency component as before (the reader may easily check that the turning point is well within the region that the "frozen approximation" is valid), and we find once again that the Bogolubov coefficient is:

$$
\left|\beta_{\{n\}}\right|^{2}=e^{-2 \pi E_{-} / \rho},
$$


which is essentially identical to the expression given in (65). This is independent of the position, despite the fact that $\int d \eta E$, and the turning point in the complex $\eta$ plane, depends on $R$. For more general scale factors the imaginary part of the integral around the turning point of $E$ will depend on $R$. In addition the time integral in the "frozen" regime has some position dependence, so that the wave function begins at $\eta=-\eta_{f}$ as a stationary state and in the "frozen" region begins to develop additional structure. Nonetheless, within the approximation we are working in, the position dependence of $\operatorname{Im} \int d \eta E$ and the perturbations of the initial wavefunction which develop as we move through the "frozen" region are subleading. One can see this by noting that

$$
\frac{\partial E}{\partial R_{n}^{k}}=\frac{n^{2}\left(C(\eta)^{2}-1\right) R_{n}^{k}}{E}
$$

thus the change in the energy arising from the change in any given oscillator coordinate will be

$$
E^{\prime} \delta R \sim \mathcal{O}(1 / \sqrt{N}) .
$$

For computing the integral $\operatorname{Im} \int d \eta E$, summing the squares of this error gives us a total error of $\mathcal{O}(1)$, compared with a lowest order answer $E_{-} / \rho$ which is $\mathcal{O}(N)$. In examining the perturbations to the stationary state wavefunction that accrue during the evolution through the "frozen" region, we can see that for a change in a single oscillator coordinate,

$$
\delta \int d \eta E \sim 2 \eta_{f} \delta E \sim \frac{|B| \ln \{|B| \rho \sqrt{N}\}}{\rho \sqrt{N}},
$$

while the integral $\int d R_{n}^{k} p_{n}^{k}$ is of $\mathcal{O}(1)$ in the $1 / N$ expansion, so that corrections to our description of the wavefunction as being frozen in this regime are indeed subleading.

After the string leaves the frozen region, the curve $\mathcal{C}_{\eta_{f}}$ will generally intersect some band of energy shells of the Hamiltonian describing the evolution beyond $\eta>\eta_{f}$, as shown in Figure 4. The curve $\mathcal{C}_{\eta>\eta_{f}}$ becomes time dependent and starts to rotate and stretch with a periodicity given by that of the final oscillator Hamiltonian.

We still need to check that our semiclassical approximation is valid, by checking Equations (53) and (54). Hamilton-Jacobi theory tells us that $\partial S / \partial q=p(q)$. Thus, the condition for Equation (53) to hold is that:

$$
\left|\frac{\partial p_{R}}{\partial R}\right|=\left|\frac{-n^{2} C(\eta)^{2} R_{n}^{k}}{p_{n}^{k}}\right| \ll\left(p_{n}^{k}\right)^{2} .
$$

This is just the usual condition for the semiclassical approximation to hold for the harmonic oscillator wavefunction (though in the relativistic case this means $\omega^{2} q \ll p^{3}$ rather than $\left.\omega^{2} q \ll p^{2}\right)$; it will work for large energies so long as we 
are sufficiently far from the turning points. The energy is large and fixed for $\eta<\eta_{f}$ in our approximation; at $\eta=\eta_{f}$ the curve $\mathcal{C}_{\eta_{f}}$ intersects a finite band of energy shells of the final Hamiltonian, all with energy larger than the initial energy if the scale factor expands (and smaller if it contracts, though so long as the change in $\omega$ is not too drastic the final range of energies should still be large). For states with oscillator modes of a given energy $E_{k, n}, p_{R}$ is given by Equation (81), and thus Equation (53) holds so long as $E_{k, n}$ is large. In our approximation this is the case; if $E_{k, n}$ was small we could treat that oscillator via the adiabatic expansion. To check Equation (54), note that

$$
\frac{\partial S}{\partial \eta}=E, \text { and } \frac{\partial^{2} S}{\partial \eta^{2}}=\frac{C_{\eta}}{C} \frac{\sum_{k, n} n^{2} C\left(\eta^{2}\right)\left(R_{n}^{k}\right)^{2}}{E} .
$$

Equation (54) holds if

$$
\frac{E_{\eta}}{E^{2}} \sim \frac{\rho}{\sqrt{N}} \ll 1,
$$

which is certainly the case. One may also work with the path integral of the wave function and check that the quadratic fluctuation operator, given by second variation of the action along the classical trajectory, contains terms of order $E$, so that the saddle point integration around the classical trajectories gives a good approximation. Recall also that it is the divergences in the determinant of this quadratic fluctuation operator which contribute to the $\beta$-function of the 2 -d $\sigma$ model; we would thus like to reiterate that although our background does not lead to a vanishing $\beta$-function, the effects of this lack of conformal invariance will appear in the determinant of the quadratic fluctuation operator, while the physics we are interested in resides in the exponential part of the wave function, $\exp \left\{i S_{\mathrm{cl} .}\right\}$.

For $\eta>\eta_{f}$, the oscillator frequency has changed and the system now has frequency $\omega_{+}$. It is no longer in a state of definite energy, however. As shown in Figure 4, the curve $\mathcal{C}_{\eta_{f}}$, which is still in our approximation identical to $\mathcal{C}_{-\infty}$, intersects many tori defined by $E=$ constant when $\omega=\omega_{+}$and so $\mathcal{C}_{\eta>\eta_{f}}$ will be time dependent. However, as we know that the wave function is stationary for $\eta<\eta_{f}$ within our approximation, it is easier to write the spatial part of the wave function as the product of Laguerre polynomials that our semiclassical wave function approximates; we may then simply decompose this wave function into energy eigenstates of the Hamiltonian for $\eta>\eta_{f}$. The reader has doubtless noted that this is essentially the sudden approximation for the spatial part of the wave functions. We may argue for this approximation more directly using the Hartree approximation for the wave functions: that is, let

$$
\Psi=\prod_{k, n} \psi_{k, n}\left(R_{n}^{k}, \phi_{n}^{k}, \eta\right)
$$

and assume that correlations in the wave function are of higher order in $1 / N$. 
We may write the wave equation applied to Equation (90)

$$
\left\{-\frac{\partial^{2}}{\partial \eta^{2}}+\sum_{k, n} \mathcal{H}_{k, n}^{\text {spatial }}\left(R_{n}^{k}, \eta\right)\right\} \Psi=0
$$

as

$$
\begin{aligned}
& \sum_{k, n}\left\{-\ddot{\psi}_{k, n} \prod_{(l, m) \neq(k, n)} \psi_{l, m}-\dot{\psi}_{k, n} \sum_{(l, m) \neq(k, n)} \dot{\psi}_{l, m} \prod_{(j, p) \neq(k, n),(l, m)} \psi_{j, p}\right. \\
& \left.+\mathcal{H}_{k, n} \psi_{k, n} \prod_{(l, m) \neq(k, n)} \psi_{l, m}\right\} \text {. }
\end{aligned}
$$

The resulting equations of motion are

$$
\left\{-\frac{\partial^{2}}{\partial \eta^{2}}-N K_{k_{0}, n_{0}}(\eta) \frac{\partial}{\partial \eta}+\mathcal{H}_{k_{0}, n_{0}}(R, \eta)\right\} \psi_{k_{0}, n_{0}}=0
$$

where

$$
N K_{k, n}(\eta)=\sum_{(l, m) \neq(k, n)} \int \psi_{l, m}^{*} \frac{\partial}{\partial \eta} \psi_{l, m} .
$$

Let us rescale $\eta \rightarrow N \eta$. The first term is now $\mathcal{O}\left(1 / N^{2}\right)$ and we shall drop it. The time dependences of $K$ and of the frequencies in $\mathcal{H}$ have the form $N \eta$, so we have a non-relativistic harmonic oscillator which varies rapidly compared to the other time scales of the problem. In particular, $C(N \eta)^{2}=A+B \tanh (N \rho \eta)$ is effectively a step function when $N \rho \gg 1$, so the sudden approximation is valid. This argument is admittedly very rough but seems to capture the right physics. A string with many oscillators highly excited will be heavy and slow; therefore, it will be nonrelativistic and any change in the spacetime, if moderate, will occur on a time scale much smaller than the response time of the string. We should note that we still need the relativistic analysis above to find the string production amplitudes, as there is no string production in the nonrelativistic approximation. 


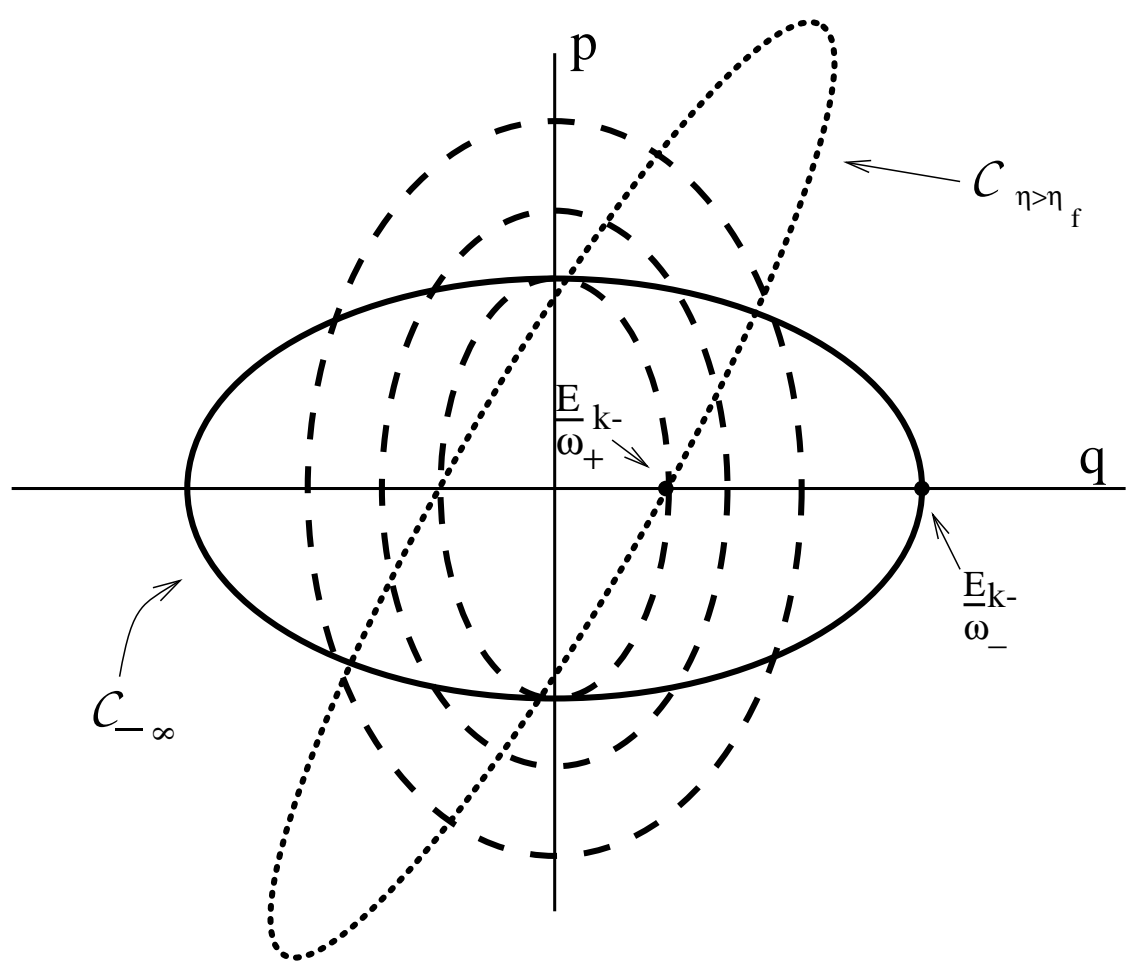

Figure 4

Evolution of one of the Fourier modes of the string. For $\eta<\eta_{f} p_{k}\left(q_{k}\right)$ is defined by the solid curve $\mathcal{C}_{-\infty}$, which is a curve of constant energy $E_{-}^{k}$ in phase space. For $\eta<\eta_{f}$ the curves of constant energy are the dashed ellipses. The dotted curve $\mathcal{C}_{\eta>\eta_{f}}$ denotes the function $p(q)$ found by evolving $\mathcal{C}_{-\infty}$ from $\eta_{f}$ to some $\eta$ using the string Hamiltonian.

We have thus shown that in this model, the number of strings per unit volume produced in a given state with energy $E_{-}$is proportional to $\exp \left\{-2 \pi E_{-} / \rho\right\}$. At sufficiently large oscillator level $N$, if the center of mass momentum is of order $1, E_{-} \approx m \approx \sqrt{N / \alpha^{\prime}}$ where $m$ is the mass. It is well known that the number of string states of mass $m$, for $m \gg 1 / \alpha^{\prime}$, can be approximated by the formula

$$
D(m) \approx m^{-b} e^{c \sqrt{\alpha^{\prime}} m}
$$

where $b$ and $c$ are pure positive numbers of order 10 and depend on the specific string theory one is using. So far we have calculated the production amplitude for an arbitrary state of a given mass regardless of whether or not it was a physical state. The density of states should automatically count physical states, so if we use it to count the total number of strings produced we should get the correct answer. Thus the total energy density produced during the expansion 
is, if we assume that our production formula holds for most string states at a given mass,

$$
\frac{\dot{\mathcal{E}}_{\text {total }}}{\text { unitvolume }} \approx \int_{0}^{\infty} d m m D(m) e^{-2 \pi m / \rho} .
$$

If we allow ourselves the luxury of extrapolating our result to expansion rates of order the inverse string scale $\sqrt{\alpha^{\prime}}$, we find that when

$$
\rho>\frac{2 \pi}{c \sqrt{\alpha^{\prime}}}
$$

the integral in (96) blows up in a manner reminiscent of the Hagedorn transition. This is the main result of this paper.

\subsection{Interpretation}

The fact that the energy produced at a critical value of the expansion rate diverges indicates to us that computing string production on a fixed background leads to a diverging rate of string production. We believe this divergence to be a sign that we have either reached some sort of phase transition in the theory, or we have neglected the effects of the backreaction of the produced string matter. We may speculate in the latter case that since the bulk of the energy is produced in very large strings as $\rho$ approaches $1 / \sqrt{\alpha^{\prime}}$, the backreaction should act to slow the expansion, as we have previously claimed should occur with a sufficient density of strings which grow with the scale factor. Since this problem will arise whenever the expansion rate is sufficiently fast, we argue that the effect of backreaction will be to sufficiently slow the expansion so that any curvature singularity, if reached, is reached at infinite proper time and that all geodesics become completeable.

\section{Speculations about anisotropically expanding models}

The arguments in the previous section are far from a rigorous solution to the problem of generic singularities, which as we have argued are very anisotropic and furthermore have no "out" region with an unambiguous definition of particle number. As for the latter, we hope that the general features of our answer in terms of the more covariant concept of the energy-momentum tensor will carry through to a calculation of this quantity in a background with diverging rates of expansion and contraction, even though the details of the calculation will be very different; in particular the essential singularity in the calculated rate of energy production seems physically well-motivated and general, being based on the fight between an exponential suppression of string production and the exponential growth of the density of states. 
If we wish to understand the further evolution of the spacetime, we need to understand how the anisotropic expansion and contraction which exists near generic singularities affects the configuration of produced strings, and we need to understand the equation of state in these anisotropic spacetimes. For example, one might expect that strings are preferentially produced with excitations in the most rapidly expanding and contracting directions, and that most of the strings produced will in the expanding case be large strings growing as the scale factor and in the contracting case be small strings with large momentum in the oscillator modes. The wave functions we have constructed are stationary states with no definite shape. To make our scenario plausible we would need to work with some set of coherent states which describe strings in definite classical configurations; since the highly massive strings are generally nonrelativistic the width of the string wave function around the peak configuration will not spread too quickly. We have not been able to detect any preference in the production of any particular configuration at our level of approximation, even in the isotropically expanding case.

As for the question of which string field modes are preferentially produced, if we examine strings which are polarized in a direction or set of directions with a given expansion rate, the number of strings produced will increase with the expansion rate in that direction. On the other hand, strings with no polarization in the expanding and contracting directions (if some spatial directions have a constant scale factor) should not be produced at all. There should be some extrapolation between these extremes, but our approximations seem to be relatively insensitive to the distribution of modes among the various polarizations. Clearly there is a considerable amount of work to do.

One natural scenario for the evolution of the spacetime is the following: as we approach the singularity, where it is approximated by the Kasner metric, string production becomes important, and we produce unstable strings preferentially polarized in the directions which are changing the most rapidly. The equation of state for the growing strings is incompatible with a constant dilaton, so the equations governing string cosmology, Equations (9), effectively describe the spacetime. As $\dot{\lambda}_{k}$ approaches the string scale, the energy becomes extremely large; the expanding directions feel a large negative pressure and the contracting directions feel a large positive pressure, and the spacetime relaxes to a nonsingular evolution.

What in fact happens will depend on the correct expressions for $\rho$ and $p$, the initial value of $\phi$, and higher order corrections to the $\beta$-function equations. Based on some simple computer simulations and qualitative arguments, we have found several possible outcomes for evolution via Equations (9). There seems to be little possibility for the rapid inflation and contraction to turn around before the expansion rate reaches unity in string units. What happens when it reaches this point depends on how we interpret the diverging integral in Equation (96). If we permit ourselves to imagine that the effect is a sufficiently large production of string matter then the expansion and contraction will suddenly reverse due 
to the large pressures we expect to arise from strings in rapidly expanding and contracting spacetimes. Once the turnaround occurs much will depend on the equation of state for less rapidly changing spacetimes. If expansion halts completely and the pressure drops to zero, then the spacetime will stabilize at a constant scale factor but the large energy density produced will cause the dilaton to grow to infinity in finite time, so that string loop effects become extremely important. We might imagine using the conjectured duality properties of string theory (see for example Sen 1994, Hull and Townsend 1995, Duff 1995, Witten 1995) at this point to relate the background to the weakly coupled background of another theory. Another scenario occurs if the string coupling is too weak when the expansion rate becomes of order unity and not enough energy is produced; the large continued contraction of the angular directions can cause the otherwise growing dilaton to return to weak coupling as we reach the singularity. Of course, in the regime we are discussing all quantities $-\rho, p_{k}, \dot{\lambda}_{k}$, and so on are of order one in string units; we can only speculate what the form of the $\beta$-function equations and the equation of state might be. In discussing the form of $\rho$ and $p$ we should note also that at the point that the expansion becomes of order unity, the singularity in the Schwarzschild geometry is reached within a single string time. It is not clear that strings can equilibrate. Nonetheless, the blow-up of the integral in Equation (96) indicates that the description of the region near the putative singularity may be drastically modified by the quantum dynamics of string field theory.

\section{Conclusions}

We have argued that in regions of rapid spatial expansion, such as the region close to a spacelike singularity, a divergent energy density of string is produced as the curvature approaches the string scale. This leads us to one of two conclusions, both of which are interesting and merit further investigation: either string theory the region close to the singularity undergoes a phase transition as the density of string approaches one string mass per string volume, or properly taking into account the back reaction of the produced strings on the spacetime pushes the singularity to infinite proper time. The answer could also be some combination of the two, or it could be that string theory is just inconsistent, although we feel that the divergence calculated has the flavor of a divergence arising from an incorrect approximation or extrapolation (i.e. from neglecting either backreaction or a phase transition at large densities), and so there is no obvious reason to call this divergence a symptom of some disease of the underlying theory.

If the backreaction becomes important in the manner we have suggested, it is an indication that we must take seriously unstable macroscopic strings as sources in the $\beta$-function equations. This not to say that solving the $\beta$-function equations gives us an inconsistent picture, but rather that high order loop effects 
become important in spacetimes with unstable strings. It would be interesting to find some sort of self-consistent way to include macroscopic strings as sources directly in the $\beta$-function equation, so that one could evolve the spacetime accordingly and see what happens to singular regions and to the singularity theorems. One might also envision trying to construct this approximation directly by resumming certain classes of loop corrections to the $\beta$-function equations.

Understanding how string theory modifies the dynamical equations of spacetime is extremely important for a resolution of the problem of black hole evaporation. A transition to a uniformly expanding region (Frolov et.al. 1990); continued nonsingular contraction of the two-spheres and expansion of the radial direction (Martinec 1995) (where "radial" refers to the spacelike direction in the interior of the black hole); or simultaneous collapse of the expanding directions are all possible depending on the density of state and the equations of motion of the background. The first two possibilities tell us that the information is carried away to inaccessible regions of spacetime; the third possibility implies a slowly-evaporating-remnant scenario, where information is brought near the horizon by the recollapse of the radial scale factor.

String production in a non-trivial background spacetime should be an approximation to the full quantum dynamics of the vacuum condensate. It may be that we have shown that it is incorrect not only in principle but in practice to separate dynamical quantum string fields and classical string backgrounds. If this is the case then we are stuck until we develop a manifestly background independent understanding of string field theory, or its nonperturbative completion.

\section{Acknowledgements}

We would like to thank Eanna Flanagan, Vivek Iyer, and Martin O'Loughlin for enlightening conversations and helpful suggestions. A.L. and E.M. are supported in part by funds provided by the DOE under grant No. DOE-FG0290ER-40560.

\section{A Matching 4d black holes to 2d black holes}

Although we have argued that classical (tree level) string theory is insufficient for understanding spacelike singularities, it would be nice to better understand the classical geometry of singularities in string theory, in order to have some confidence in our starting point and to sharpen some of the arguments in the Introduction regarding the existence of singularities in string theory. We would like to repeat and extend some arguments made by Giddings et.al. (1993, 1994) concerning the worldsheet renormalization group flow of the $\sigma$-model, here without the benefit of any gauge field or axion hair to stabilize the shrinking twospheres. 
Close to the singularity the spacetime metric should look like

$$
d s^{2}=-d T^{2}+a^{2}(T) d \rho^{2}+r^{2}(T) d \Omega^{2} .
$$

In the classical geometry we approach the singularity in finite time as the metric in the $\rho$ direction blows up, and we must go beyond the lowest order solution to the $\beta$-function equations. It is well-known that the $S^{2}$ part of the $\sigma$-model should flow to a trivial $c=0$ theory. Cecotti and Vafa (1992) have computed the Zamolodchikov metric along the entire renormalization group flow of the supersymmetric $C P^{1}=S^{2}$ model and found the metric to be well defined, even as $r^{2}$ becomes negative (see also Witten 1993). As the radius gets small and passes through zero the theory masses up and becomes trivial, with the soliton masses increasing as $m=8 \exp \left(-r^{2} / 2\right)$. Once the radius is sufficiently negative, the $T, \rho$ plane will have excess central charge; a natural fixed point of the renormalization group flow is the $S L(2, \mathcal{R}) / U(1)$ black hole. This would mean that the velocity of the transverse stretching direction dominates the $\beta$ function for $a$. One might convince oneself of this by adding a potential term to the spacetime action simulating the effects of string instantons wrapping around the $S^{2}$ direction (as do Giddings et.al. (1994)). This potential should be of the form

$$
V(R, \phi)=e^{-2 \phi-2 r^{2} / \alpha^{\prime}}
$$

(Giddings et.al. 1994) where $\phi$ is the dilaton. This is a function of the scale factor which is of order unity at zero radius: it cannot compete with the large $\dot{\lambda}$ terms present near a velocity-dominated singularity.

This is an attractive scenario, but it has not been proven. Exact results such as those of Cecotti and Vafa (1992) do not help us much. The contribution of the $S^{2}$ degrees of freedom to the dilaton $\beta$-function should be given by the Zamolodchikov $c$-function of the $S^{2}$ theory (Fradkin and Tseytlin 1985, Callan et.al. 1985, Polyakov 1986, 1987), which is only simply related to the Zamolodchikov metric at RG fixed points (Zamolodchikov 1986). Even if we had this information, cross-couplings between $a$ and $r$ would make the calculation exceedingly difficult.

We can also try to work backwards from the singularity, starting with the WZW black hole at small small $r$, (where $r$ is the timelike direction inside the horizon, and $t$ is the spacelike coordinate), times the $S^{2}$ theory close to its trivial infrared fixed point; with these initial conditions, the velocity terms coming from the coupling of the $S^{2}$ are small and we have some intuition for the evolution of the $\beta$-function equations. Here we would use the following procedure. Begin with a spacelike slice $\Sigma$ in this region. Given the mass of the WZW black hole, a starting point inside the horizon, and a starting point in the RG trajectory of the $S^{2}$ theory, we have a set of initial conditions for evolving the $\beta$-function equations of the $\sigma$-model away from the singularity in the radial direction. We may do this, for example, by using the well-known analogy between the $\beta$-function equations in spacetime and the RG flow of the spatial part of the 
$\sigma$-model, where in the latter picture we would gravitationally dress the spatial $\sigma$-model with appropriate functions of the Liouville mode (Polchinski 1989, Das et.al. 1989, Banks and Lykken 1990). In the beginning of the radial evolution we find that the spacetime is some small perturbation of the WZW black hole coupled to the $S^{2}$ theory moving away from the trivial fixed point. If we begin with the $S^{2}$ theory close enough to this fixed point, as we pass through the horizon at $r=r_{h}$ the geometry will still be described by the WZW black hole times the massive $S^{2}$ theory with the mass slowly decreasing, and the $r-t$ theory approaches the asymptotically flat linear dilaton vacuum. At some point $r=r_{c}$ the $S^{2}$ theory will begin crossing over to behavior characterized by the ultraviolet, asymptotically free fixed point and we should enter an asymptotically flat regime with a constant dilaton (since there is no longer any excess central charge to feed a linear dilaton solution). At this point, by Birkhoff's theorem, any deviations from the Minkowski metric should be that arising from the Schwarzschild solution at large radius. If we evolve the geometry from $\Sigma$ towards the singularity, as long as the $S^{2}$ theory sufficiently close to its IR fixed point, we expect to reach the singularity of the WZW black hole, in finite time, since the effect of the flow of the $S^{2}$ theory should be minimal. The geometry is then that of a long throat with an effectively 2-dimensional geometry, terminating at one end at a $2 \mathrm{~d}$ black hole singularity and opening out at the radius $r=r_{c}$, well outside the horizon of the $2 \mathrm{~d}$ theory, into an asymptotically flat 4-dimensional spacetime. It may be that when we reach $r_{c}$ the crossover forces us into some other fixed point, but we believe this to be unlikely.

Now let us move in the space of $c=4$ conformal field theories by moving the initial position of the $S^{2}$ theory farther away from its trivial fixed point. This should have the effect of increasing the initial velocity of the coupling of the $S^{2}$ theory to $r$, which making cross-coupling terms between the radial and angular directions more important. We then expect $r_{c}$ to approach $r_{h}$; eventually along this marginal line, $r_{h}$ enters the asymptotically flat regime, unless we are driven by the crossover behavior to some other fixed point. Since at this point, the velocity terms coming from $S^{2}$ at $\Sigma$ should be fairly large, we no longer expect the region near the singularity to look like the WZW black hole; the RG flow might push the WZW singularity off to infinite distance, but we expect instead that we have some standard velocity-dominated singularity, based on our previous arguments.

\section{References}

Amati D and Klimcik C 1989 Phys. Lett. B 219443

Aspinwall P S, Greene B R, and Morrison D R 1993 Phys. Lett. B 303249

Aspinwall P S, Greene B R, and Morrison D R 1994 Nucl. Phys. B 416414 
Audretsch J 1979 J. Phys. A 121189

Banks T 1994 Lectures on Black Holes and Information Loss (Lectures from the Spring School on Supersymmetry, Supergravity and Superstrings (Trieste)) hep-th/9412131

Banks T and Lykken J 1990 Nucl. Phys. B 331173

Belinskii V A, Khalatnikov I M, and Lifshitz E M 1970 Adv. Phys. 19525

Belinskii V A, Khalatnikov I M, and Lifshitz E M 1982 Adv. Phys. 31639

Bernard C and Duncan A 1977 Ann. Phys, NY 107201

Berry M V 1981 Chaotic Behavior of Deterministic Systems, Les Houches summer school proceedings (Amsterdam, North-Holland)

Berry M V and Balazs N 1979 J. Phys. A 12625

Birrell N D and Davies P C W 1982 Quantum Fields in Curved Space (Cambridge, UK: Cambridge University Press)

Callan C G, Friedan D, Martinec E J, and Perry M P 1985 Nucl. Phys. B 262

Callan C G, Giddings S B, Harvey J A, and Strominger A 1992 Phys. Rev. D $45 \mathrm{R} 1005$

Callan C G and Khuri R R 1991 Phys. Lett. B 261363

Callan C G, Lovelace C, Nappi C R, and Yost S A 1987 Nucl. Phys. B 288 525

Cecotti S and Vafa C 1992 Phys. Rev. Lett. 68903

Dabholkar A and Harvey J A 1989 Phys. Rev. Lett. 63719

Dabholkar A, Gibbons G, Harvey J A, and Ruiz-Ruiz F 1990 Nucl. Phys. B 34033

Das S, Naik S, and Wadia S 1989 Mod. Phys. Lett. A 41033

de Vega H and Sanchez N 1992 Phys. Rev. D 452783

DeWitt B S 1957 Rev. Mod. Phys. 29377

Dijkgraaf R, Verlinde H, and Verlinde E 1992 Nucl. Phys. B 371269

Dixon L J, Harvey J A, Vafa C, and Witten E 1985 Nucl. Phys. B 261678

Dixon L J, Harvey J A, Vafa C, and Witten E 1986 Nucl. Phys. B 274285

Duff M 1995 Nucl. Phys. B 44247

Eckhardt C 1930 Phys. Rev. 351303

Epstein H, Gaser V, and Jaffe A 1965 Nuovo Cimento 361016

Fischler W and Susskind L 1986 Phys. Lett. B 171 383, Phys. Lett. B 173 262

Fradkin E S and Tseytlin A A 1985 Nucl. Phys. B 2611 
Friedan D 1985 Phys. Lett. B 162102

Frolov V P, Markov M A, and Mukhanov V F 1990 Phys. Rev. D 41383

Fulling S A 1989 Aspects of Quantum Field Theory in Curved Spacetimes (Cambridge, UK: Cambridge University Press)

Gasperini M, Sanchez N, and Veneziano G 1991a Int. J. Mod. Phys. A 6 3853

Gasperini M, Sanchez N, and Veneziano G 1991b Nucl. Phys. B 364365

Gauntlett J P, Harvey J A, Robinson M M, and Waldram D 1994 Nucl. Phys. B 411461

Giddings S B, Polchinski J, and Strominger A 1993 Phys. Rev. D 485784

Giddings S B, Harvey J A, Polchinski J G, Shenker S H, and Strominger A 1994 Phys. Rev. D 506422

Gross D J and Mende P F 1987 Phys. Lett. B 197129

Gross D J and Mende P F 1988 Nucl. Phys. B 303407

Hawking S W 1967 Proc. Roy. Soc. (London) A 300182

Hawking S W 1974 Nature (London) 24830

Hawking S W and Penrose R 1970 Proc. Roy. Soc. (London) A 314529

Horowitz G T and Myers R 1995 The value of singularities UCSB preprint UCSBTH-95-6, gr-qc/9503062

Horowitz G T and Steif A R 1990, Phys. Rev. Lett. 64 260, Phys. Rev. D 42 1950

Hull C M and Townsend P K 1995 Nucl. Phys. B 438109

Johnson C V 1994 Phys. Rev. D 504032

Khuri R R 1993 Phys. Lett. B 307 298, Nucl. Phys. B 403 335, Phys. Rev. D 482823

Landau, L D and Lifshitz E M 1977, Quantum Mechanics: non-relativistic theory, 3rd. ed. (NY: Pergamon)

Lifshitz E M and Khalatnikov I M 1963 Adv. Phys. 12185

Lovelace C 1986 Nucl. Phys. B 273413

Lowe D and Strominger A 1994 Phys. Rev. Lett. 731468

Martinec E J 1995 Class. Quant. Grav. 12941

Maslov V P and Fedoriuk M V 1965, Semiclassical Approximation in Quantum Mechanics (Dordrecht: Reidel) (original Russian edition 1965)

Migdal A B 1977 Qualitative Methods in Quantum Theory (Reading MA: W.A. Benjamin)

Nappi C and Witten E 1992 Phys. Lett. B 293309 
Penrose R 1965 Phys. Rev. Lett. 1457

Peremolov A M and Popov V S 1969 Sov. Phys. JETP 39738

Poisson E and Israel W 1988 Class. Quant. Grav. 5 L201

Polchinski J 1989 Nucl. Phys. B 324123

Polyakov A M 1986 Phys. Scripta T15 191

Polyakov A M 1987 Gauge Fields and Strings (New York: Harwood Academic)

Pokrovskii V I and Khalatnikov I M 1961 Sov. Phys. JETP 131207

Sanchez N and Veneziano G 1990 Nucl. Phys. B 333253

Sauter 1932 Z Phys. 73547

Schoen T and Yau S-T 1983 Commun. Math. Phys. 90575

Sen A 1985 Phys. Rev. D 32 2102, Phys. Rev. Lett. 551846

Sen A 1994 Int. J. Mod. Phys. A 93707

Strominger A 1995 Les Houches Lectures on Black Holes, Lectures the Les Houches Summer School, "Fluctuating geometries in statistical mechanics and field theory", Les Houches, France, hep-th/9501071

Tseytlin A A 1990 Phys. Lett. B 251530

Tseytlin A A 1992 Class. Quant. Grav. 9979

Tseytlin A A and Vafa C 1992 Nucl. Phys. B 372443

Veneziano G 1991 Phys. Lett. B 265287

Witten E 1991 Phys. Rev. D 44314

Witten E 1993 Nucl. Phys. B 403159

Witten E 1995 Nucl. Phys. B 44385

Zamolodchikov A B 1986 JETP Lett. 43730 